\title{
Über die Ökonomischen Ursachen von Bürgerkriegenı
}

\section{Paul Collier und Anke Hoeffler ${ }^{2}$}

Paul Collier, Development Research Group, World Bank, 1818 H Street, N.W., MSN MC3-304, Washington, D.C. 20433, U.S.A.

Anke Hoeffler, Peace Research Institute Oslo und Centre for the Study of African Economies, University of Oxford, 21 Winchester Rd, Oxford OX2 6NA, anke.hoeffler@economics.ox.ac.uk

Wir untersuchen die Ursachen von Bürgerkriegen wobei wir einen neuen Datensatz verwenden, der die Jahre 1960 bis 1999 umfasst. Dabei testen wir zwei Theorien: Einerseits können Bürgerkriege durch atypische Möglichkeiten erklärt werden, z.B. durch gute Finanzierungsmöglichkeiten der Rebellen und niedrige Kosten der Rekrutierung. Andererseits werden häufig Leidensfaktoren, z.B. politische Unterdrückung, Ungleichverteilung sowie ethnischer und religiöser Hass, als Ursachen von Bürgerkriegen zitiert. Unsere empirischen Ergebnisse zeigen, dass die Theorie der atypischen Möglichkeiten einen besseren Erklärungsansatz bietet als die Leid-Theorie. Reichtum an natürlichen Ressourcen und eine große Diaspora, definiert als der Anteil der Bevölkerung der im Ausland lebt, erhöhen das Bürgerkriegsrisiko erheblich, denn beide Faktoren erleichtern die Finanzierung einer Rebellion. Entgegen der häufig vertretenen Meinung, dass ethnische und religiöse Diversität das Bürgerkriegsrisiko erhöht, kommen wir zu dem Ergebnis, dass ein hoher Grad an Diversität dieses Risiko verringert, da es schwieriger ist, eine Rebellenorganisation aufzubauen. Unsere Ergebnisse sind robust in Hinsicht auf Ausreißer, alternative Variablendefinitionen und Variationen in der Schätzmethode.

1 Dieser Beitrag fasst die Erkenntnisse früherer Arbeiten zusammen, insbesondere die Ergebnisse des Arbeitspapieres "Greed and Grievance in Civil War", CEPR Working Paper (angenommen).

2 Paul Collier ist der Direktor der Forschungsabteilung der Weltbank. Dieser Beitrag ist keine offizielle Stellungnahme der Weltbank, sondern gibt lediglich die persönliche Meinung der Autoren wider. Anke Hoeffler möchte sich bei der Weltbank und dem Norwegischen Forschungsrat (NFR) für ihre finanzielle Unterstützung bedanken. Rückfragen an: anke.hoeffler@economics.ox.ac.uk 


\section{Einleitung ${ }^{3}$}

Seit der Tagung in Würzburg, auf der dieser Beitrag vorgestellt wurde, wurden die USA Opfer von bislang unvorstellbaren terroristischen Angriffen. Leider zeigen diese Terrorangriffe wie aktuell das Thema der sozialen Kohäsion ist. Seit einiger Zeit sind Kriege weniger durch Kämpfe zwischen feindlichen Truppen gekennzeichnet, sondern durch das Töten der Zivilbevölkerung. In zunehmendem Maße erleben wir eine Globalisierung von Konflikten, und die Welt erscheint uns weniger sicher als vor dem 11. September 2001. Oft ist Terrorismus der Beginn oder die Fortsetzung eines Bürgerkrieges mit anderen Mitteln. In diesem Beitrag wollen wir die Ursachen von Bürgerkriegen untersuchen und uns besonders der Frage widmen, ob solche Konflikte auch ökonomische Ursachen haben.

Bürgerkriege sind heute wesentlich häufiger als Kriege zwischen Nationalstaaten: im Jahr 2000 gab es weltweit 25 schwere bewaffnete Konflikte, darunter 23 Bürgerkriege. ${ }^{4}$

Wir entwickeln ein ökonometrisches Modell, mit dessen Hilfe wir den Ausbruch eines Bürgerkrieges analysieren können. Unser Modell berücksichtigt neben ökonomischen Variablen auch sozio-politische, geographische und historische Determinanten. Im nachfolgenden Abschnitt unseres Beitrages beschreiben wir Daten für 78 Bürgerkriege über einen Zeitraum von 1960 bis 1999 und entwickeln zwei Erklärungsansätze. Eine Theorie basiert auf der Motivation der Rebellen und untersucht den Einfluss von objektiv messbaren Leidfaktoren, wie z.B. politische Unterdrückung und Ungleichverteilung, auf das Bürgerkriegsrisiko. Der zweite Erklärungsansatz analysiert den Zusammenhang des Bürgerkriegsrisikos und der ökonomischen Möglichkeit der Kriegsführung, hierbei konzentrieren wir uns auf die Finanzierung des Konfliktes und die Opportunitätskosten der Rebellen. Der dritte Abschnitt stellt die empirischen Ergebnisse der beiden konkurrierende Modelle dar. Statistische Tests zeigen, dass wir keines der beiden Modelle völlig ablehnen können, weswegen wir eine Synthese der beiden Erklärungsansätze vorschlagen. Im darauffolgenden Abschnitt führen wir eine Reihe von zusätzlichen Tests durch, um die Robustheit unserer Ergebnisse zu überprüfen. Im letzten Abschnitt fassen wir die Ergebnisse zusammen. Wir zeigen, dass das Möglichkeiten-Modell eine bessere Erklärung für Bürgerkriege darstellt als Motivation. Eine besonders wichtige erklärende Variable ist die Abhängigkeit von Primärproduktexporten. Primärprodukte stellen eine lukrative Einkommensquelle für Rebellenorganisationen dar. Ob diese

3 Wir möchten uns bei Alberto Alesina und einem anonymen Gutachter für Kommentare bedanken. Frühere Versionen dieses Papiers wurden in CERDI, NYU, LSE, Princeton, Oxford, Würzburg, Barcelona, Lissabon und an der Weltbank vorgetragen. Wir möchten uns bei allen Seminarteilnehmern für Kommentare und Verbesserungsvorschläge bedanken, ganz besonders bei Todd Sandler, der uns ausführliche Kommentare schickte.

$4 \quad$ Stockholm Peace Research Institute (SIPRI), Yearbook 2001. 
Einnahmen den Bürgerkrieg direkt motivieren oder ob sie nur Mittel zum Erreichen anderer Ziele darstellen, können wir allerdings nicht im Rahmen dieser Studie untersuchen.

\section{Bürgerkriege: Empirische Fakten und Erklärungsansätze}

\section{Präferenzen, Möglichkeiten und Wahrnehmung}

Wir definieren Bürgerkriege als interne Konflikte mit einem Minimum von 1000 Todesopfern pro Jahr. Hierbei zählen Militär- und Zivilopfer, die aufgrund von direkter Kampfeinwirkung starben, nicht aber die Opfer von kriegsbedingten Seuchen oder Hungersnöten. Von diesen Opfern wurden mindestens fünf Prozent von organisierten Rebellen getötet. Diese Definition schliesst u.a. Massaker und Pogrome aus und geht auf Singer und Small $(1982,1994)$ zurück. Wir verwenden eine erweiterte Version ihres Datensatzes, der 161 Länder über einen Zeitraum von 1960 bis 1999 erfasst. In diesem lassen sich 78 Bürgerkriege identifizieren, die in Tabelle 1 dargestellt werden. Unser Ziel ist es, den Ausbruch dieser Bürgerkriege zu analysieren.

Hirshleifer $(1995,2001)$ klassifiziert die verschiedenen Konfliktursachen in drei Kategorien: Präferenzen, Möglichkeiten und Wahrnehmungen. Typischerweise konzentrieren sich Politikwissenschaftler auf die Analyse von Präferenzen in der Konfliktforschung. Rebellion wird als politischer Protest interpretiert, ausgelöst durch atypisches starkes Leid aufgrund von Ungleichheit, Unterdrückung oder ethnisch-religiösem Antagonismus. Die wenigen ökonomischen Beiträge zum Thema Konfliktforschung hingegen konzentrieren sich auf die Analyse von Möglichkeiten. Die Arbeiten von Grossman (1991, 1999) sind typisch für diesen Erklärungsansatz. In seinem Modell wird Rebellion als eine Firmenaktivität beschrieben, Rebellen plündern, um Gewinne zu erzielen, d.h. wir können Bürgerkriegsorganisationen nicht von Banditen oder Piraten unterscheiden (Grossman, 1999: 269). Gier motiviert solche Rebellionen, Präferenzen werden nicht berücksichtigt. Allerdings wird Gier als ein universelles Phänomen interpretiert und nach dem Machiavelli-Theorem (Hirshleifer, 2001: 10-11) wird jede gewinnversprechende Möglichkeit wahrgenommen. Rebellion wird daher durch atypische Umstände erklärt, die profitable Möglichkeiten erzeugen. Wir können somit festhalten, dass sich Politikwissenschaftler und Ökonomen auf verschiedene Erklärungsansätze in der Konfliktforschung konzentrieren. Die Motivation der Rebellen ist entweder Leid oder Gier, und ein Konflikt wird entweder durch atypisches Leid oder atypische Möglichkeiten erklärt. 
Tabelle 1: Bürgerkriegsausbrüche

\begin{tabular}{|c|c|c|c|}
\hline Land & $\begin{array}{l}\text { Beginn des } \\
\text { Krieges }\end{array}$ & $\begin{array}{l}\text { Ende des } \\
\text { Krieges }\end{array}$ & Früherer Krieg \\
\hline Äthiopien & $07 / 74$ & $05 / 91$ & \\
\hline Afghanistan & $04 / 78$ & $02 / 92$ & \\
\hline Afghanistan & $05 / 92$ & andauernd & $*$ \\
\hline Algerien & $07 / 62$ & $12 / 62$ & $*$ \\
\hline Algerien & $05 / 91$ & andauernd & $*$ \\
\hline Angola & $02 / 61$ & $11 / 75$ & \\
\hline Angola & $11 / 75$ & $05 / 91$ & $*$ \\
\hline Angola & $09 / 92$ & andauernd & $*$ \\
\hline Aserbeidschan & $04 / 91$ & $10 / 94$ & \\
\hline Birma/Myanmar & 68 & $10 / 80$ & $*$ \\
\hline Birma/Myanmar & $02 / 83$ & $07 / 95$ & $*$ \\
\hline Bosnien & $03 / 92$ & $11 / 95$ & \\
\hline Burundi & $04 / 72$ & $12 / 73$ & \\
\hline Burundi & $08 / 88$ & $08 / 88$ & $*$ \\
\hline Burundi & $11 / 91$ & andauernd & $*$ \\
\hline China & $01 / 67$ & $09 / 68$ & $*$ \\
\hline Dominikan. Rep. & $04 / 65$ & $09 / 65$ & \\
\hline El Salvador & $10 / 79$ & $01 / 92$ & \\
\hline Georgien & $06 / 91$ & $12 / 93$ & \\
\hline Guatemala & $07 / 66$ & $07 / 72$ & $*$ \\
\hline Guatemala & $03 / 78$ & $03 / 84$ & $*$ \\
\hline Guinea-Bissau & $12 / 62$ & $12 / 74$ & \\
\hline Indien & $08 / 65$ & $08 / 65$ & $*$ \\
\hline Indien & 84 & 94 & $*$ \\
\hline Indonesien & $06 / 75$ & $09 / 82$ & $*$ \\
\hline Irak & $09 / 61$ & $11 / 63$ & $*$ \\
\hline Irak & $07 / 74$ & $03 / 75$ & $*$ \\
\hline Irak & $01 / 85$ & $12 / 92$ & $*$ \\
\hline Iran & $03 / 74$ & $03 / 75$ & \\
\hline Iran & $09 / 78$ & $12 / 79$ & $*$ \\
\hline Iran & $06 / 81$ & $05 / 82$ & $*$ \\
\hline Jordanien & $09 / 71$ & $09 / 71$ & \\
\hline Kambodscha & $03 / 70$ & $10 / 91$ & $*$ \\
\hline Kolumbien & $04 / 84$ & andauernd & $*$ \\
\hline Kongo & 97 & $10 / 97$ & \\
\hline Laos & $07 / 60$ & $02 / 73$ & $*$ \\
\hline Libanon & $05 / 75$ & $09 / 92$ & $*$ \\
\hline Liberia & $12 / 89$ & $11 / 91$ & \\
\hline Liberia & $10 / 92$ & $11 / 96$ & $*$ \\
\hline Marokko & $10 / 75$ & $11 / 89$ & $*$ \\
\hline Mosambik & $10 / 64$ & $11 / 75$ & \\
\hline Mosambik & $07 / 76$ & $10 / 92$ & $*$ \\
\hline Nicaragua & $10 / 78$ & $07 / 79$ & \\
\hline Nicaragua & $03 / 82$ & $04 / 90$ & \\
\hline Nigeria & $01 / 66$ & $01 / 70$ & \\
\hline Nigeria & $12 / 80$ & $08 / 84$ & $*$ \\
\hline Pakistan & $03 / 71$ & $12 / 71$ & \\
\hline Pakistan & $01 / 73$ & $07 / 77$ & \\
\hline Peru & $03 / 82$ & $12 / 96$ & \\
\hline Philippinen & $09 / 72$ & $12 / 96$ & $*$ \\
\hline Rumänien & $12 / 89$ & $12 / 89$ & \\
\hline
\end{tabular}


Fortsetzung Tabelle 1

\begin{tabular}{|l|l|l|l|}
\hline Russland & $12 / 94$ & $08 / 96$ & $*$ \\
\hline Russland & $09 / 99$ & andauernd & $*$ \\
\hline Ruanda & $11 / 63$ & $02 / 64$ & $*$ \\
\hline Ruanda & $10 / 90$ & $07 / 94$ & $*$ \\
\hline Sierra Leone & $03 / 91$ & $11 / 96$ & $*$ \\
\hline Sierra Leone & $05 / 97$ & $07 / 99$ & \\
\hline Simbabwe & $12 / 72$ & $12 / 79$ & \\
\hline Somalia & $04 / 82$ & $05 / 88$ & $*$ \\
\hline Somalia & $05 / 88$ & $12 / 92$ & $*$ \\
\hline Sri Lanka & $04 / 71$ & $05 / 71$ & \\
\hline Sri Lanka & $07 / 83$ & andauernd & $*$ \\
\hline Sudan & $10 / 63$ & $02 / 72$ & \\
\hline Sudan & $07 / 83$ & andauernd & $*$ \\
\hline Tadschikistan & $04 / 92$ & $12 / 94$ & \\
\hline Tschad & $03 / 80$ & $08 / 88$ & \\
\hline Türkei & $07 / 91$ & andauernd & \\
\hline Uganda & $05 / 66$ & $06 / 66$ & \\
\hline Uganda & $10 / 80$ & $04 / 88$ & $*$ \\
\hline Vietnam & $01 / 60$ & $04 / 75$ & $*$ \\
\hline Yemen, Arab. Rep. & $11 / 62$ & $09 / 69$ & $*$ \\
\hline Yemen, Volksrep. & $01 / 86$ & $01 / 86$ & $*$ \\
\hline Yemen & $05 / 90$ & $10 / 94$ & $*$ \\
\hline Yugoslavien & $04 / 90$ & $01 / 92$ & \\
\hline Yugoslavien & $10 / 98$ & $04 / 99$ & \\
\hline Zaire/DRC & $07 / 60$ & $09 / 65$ & \\
\hline Zaire/DRC & $09 / 91$ & $12 / 96$ & \\
\hline Zaire/DRC & $09 / 97$ & $09 / 99$ & \\
\hline Zypern & $07 / 74$ & $08 / 74$ & $*$ \\
\hline & & & $*$ \\
\hline
\end{tabular}

Bemerkung: Frühere Bürgerkriege beziehen sich auf die Periode 1945-1994.

Allerdings können Rebellen eine falsche Wahrnehmung von Leid und Möglichkeiten haben. Rebellionen finden statt, wenn die Gewinnmöglichkeit aus dem Konflikt überschätzt wurde, eine Analogie zum "winners' curse". Allerdings werden diese Rebellionen in den meissten Fällen zusammenbrechen, bevor sie unseren kritischen Schwellenwert erreichen. Wenn übertriebenes Leid der Auslöser eines Konfliktes ist, werden die Kämpfe nicht das Leid mindern, sondern im Gegensatz echtes Leid verursachen. In allen Ländern gibt es gesellschaftliche Gruppen, die ihr Leid übertreiben, daher bietet das Leidmotiv keinen spezifischen Erklärungsansatz. Bürgerkriegsländer müssen sich daher von anderen Ländern dahingehend unterscheiden, dass Rebellionen durch atypische Möglichkeiten realisiert werden können.

In unserer empirischen Arbeit können wir nicht zwischen Rebellionen, die durch Gier oder übertriebenes Leid motiviert sind unterscheiden, da wir Motivation nicht messen können. Allerdings gibt es gemeinsame Faktoren, die Kriege ermöglichen. Wir besprechen nun die Variablen, mit denen wir Möglichkeiten und objektive Leidfaktoren erfassen können. 


\section{Messwerte für Möglichkeiten/Durchführbarkeit}

Wir diskutieren zuerst atypische Möglichkeiten der Finanzierung von Bürgerkriegen und konzentrieren uns auf drei Finanzquellen: Raub von natürlichen Ressourcen, Zuwendungen von Diasporen und feindlichen Regierungen.

Eine ausführliche Diskussion über den Raub natürlicher Ressourcen, z.B. Diamanten in Westafrika, Hölzern in Kambodscha und Kokain in Kolumbien, findet sich in Klare (2001). In Tabelle 2 messen wir Ressourcenreichtum als den Anteil von Primärproduktexporten am Bruttosozialprodukt (BSP). Unser Panel umfasst Informationen über acht Perioden, 1960-64, 1965-69, ..., 1995-99 für 161 Länder. Wir erfassen die meisten Erklärungsvariablen am Anfang der Periode und untersuchen, ob ein Bürgerkrieg in den folgenden fünf Jahren ausbrach, d.h. unsere Analyse beruht darauf, ob sich die erklärenden Variablen für Bürgerkriegsepisoden systematisch von denen der Friedensperioden unterscheiden. Die deskriptiven Statistiken unterstützen die Möglichkeits-Theorie nicht, Bürgerkriegsausbrüche sind durch einen etwas geringeren Anteil von Primärproduktexporten gekennzeichnet als Friedensperioden. Allerdings ist die Streuung der Variablen sehr unterschiedlich, für Friedensperioden ist die Standardabweichung mit 0.19 fast doppelt so hoch wie für Bürgerkriegsausbrüche (0.11). Mit anderen Worten: Friedensperioden haben entweder sehr niedrige oder sehr hohe Werte, während die Variable für die Bürgerkriegsausbrüche enger um den Mittelwert verteilt ist. Es ist vorstellbar, dass Länder, die durch einen hohen Anteil an Primärproduktexporten gekennzeichnet sind, z.B. Saudi Arabien, besonders hohe Steuereinnahmen erzielen und somit ein relativ hohes Verteidigungsbudget haben. Dies würde eine Rebellion militärisch unmöglich machen. Dieser Budgeteffekt könnte den Raubeffekt ausgleichen, wodurch der Gesamteffekt von Primärproduktexporten auf das Bürgerkriegsrisiko möglicherweise nicht-linear ist. Weiterhin werden Primärprodukte mit Merkmalen assoziert, die Bürgerkriege mitverursachen, wie z.B. ineffiziente Bürokratien und wirtschaftliches Missmanagement aufgrund von rent-seeking und erhöhter Korruption (Sachs und Warner, 1995, 2000). Es ist daher auch vorstellbar, dass jeder Anstieg des Bürgerkriegsrisikos eine Reaktion der Rebellen gegen eine schlechte Regierung ist und nicht durch finanzielle Möglichkeiten bestimmt wird.

Eine zweite mögliche Finanzquelle der Rebellen sind Diasporen. Unter Diaspora verstehen wir die Staatsangehörigen, die ausserhalb ihres Landes leben. Die Studie über die Rolle von Diasporen von Angoustures und Pascal (1996) zeigt z.B. deutlich, wie die Tamil Tigers in Sri Lanka von Tamilen in Nordamerika unterstützt werden. Wir messen die Größe der Diaspora durch die Anzahl der Einwanderer in die USA aus US-Volkszählungsdaten. Der Nachteil dieser Variablen ist die Vernachlässigung der Diaspora in anderen Ländern, der Vorteil hingegen ist, dass wir mit relativ homogenen Daten arbeiten, denn alle von uns erfassten Diasporen leben im gleichen legalen, organisatorischen und 
ökonomischen Umfeld. Wir dividieren die Zahl der Einwanderer in die USA durch die Gesamtbevölkerung des Herkunftslandes. Die Mittelwerte in Tabelle 2 unterstützen nicht die Möglichkeits-Theorie, da die Diaspora erheblich kleiner für die Bürgerkriegsstichprobe ist.

Tabelle 2: Deskriptive Statistiken (Mittelwerte)

\begin{tabular}{|c|c|c|c|}
\hline & $\begin{array}{l}\text { Stichprobe } \\
(n=1167)\end{array}$ & $\begin{array}{l}\begin{array}{l}\text { Friedens- } \\
\text { episoden }\end{array} \\
(n=1089)\end{array}$ & $\begin{array}{l}\text { Bürger- } \\
\text { kriegs- } \\
\text { episoden } \\
(\mathrm{n}=78)\end{array}$ \\
\hline $\begin{array}{l}\text { Bürgerkriegsausbrüche } \\
\text { (Prozent) }\end{array}$ & 6.7 & 0 & 100 \\
\hline $\begin{array}{l}\text { Primärproduktexporte/BSP } \\
\text { (Prozent) }\end{array}$ & 16.8 & 16.9 & 14.9 \\
\hline $\begin{array}{l}\text { Pro-Kopf Einkommen } \\
\text { (konst. US\$, Basisjahr 1985) }\end{array}$ & 4061 & 4219 & 1645 \\
\hline $\begin{array}{l}\text { Diaspora } \\
\text { (Prozent der Bevölkerung, die in den USA lebt) }\end{array}$ & 1.7 & 1.8 & 0.4 \\
\hline $\begin{array}{l}\text { Schulbesuch junger Männer (Prozent der Männer } \\
\text { die weiterführende Schulen besuchen) }\end{array}$ & 43.4 & 44.4 & 30.3 \\
\hline $\begin{array}{l}\text { Einkommenswachstum } \\
\text { (Prozent, Durchschnitt der letzten fünf Jahre) }\end{array}$ & 1.6 & 1.7 & -0.2 \\
\hline $\begin{array}{l}\text { Frühere Kriege } \\
\text { (Prozent der Länder mit historischen Konflikten) }\end{array}$ & 20.8 & 18.5 & 53.8 \\
\hline $\begin{array}{l}\text { Friedensperiode } \\
\text { (Monate) }\end{array}$ & 326.6 & 334.2 & 221.0 \\
\hline $\begin{array}{l}\text { Bewaldung } \\
\text { (Prozent der Landesfläche) }\end{array}$ & 31.1 & 31.3 & 27.8 \\
\hline $\begin{array}{l}\text { Bergiges Terrain } \\
\text { (Prozent der Landesfläche) }\end{array}$ & 15.8 & 15.2 & 24.9 \\
\hline $\begin{array}{l}\text { Geographische Verteilung der Bevölkerung } \\
\text { (Gini-Koeffizient) }\end{array}$ & 0.57 & 0.57 & 0.60 \\
\hline $\begin{array}{l}\text { Bevölkerungsdichte } \\
\left(\text { Einwohner pro } \mathrm{km}^{2}\right)\end{array}$ & 150 & 156 & 62 \\
\hline $\begin{array}{l}\text { Urbanisierungsgrad } \\
\text { (Prozent der Bevölkerung, die in Städten lebt) }\end{array}$ & 45.1 & 46.0 & 32.7 \\
\hline $\begin{array}{l}\text { Ethnische Fragmentierung } \\
\text { (Index 0-100) }\end{array}$ & 39.6 & 38.6 & 52.6 \\
\hline $\begin{array}{l}\text { Religiöse Fragmentierung } \\
\text { (Index 0-100) }\end{array}$ & 36.1 & 36.0 & 37.7 \\
\hline $\begin{array}{l}\text { Ethnische Polarisierung } \alpha=1.6 \\
\text { (Index 0-0.165) }\end{array}$ & 0.078 & 0.077 & 0.076 \\
\hline $\begin{array}{l}\text { Ethnische Dominanz (Prozent der Länder in denen } \\
\text { die grösste ethn. Gruppe } 45-90 \% \text { ausmacht) }\end{array}$ & 46.5 & 46.5 & 45.2 \\
\hline $\begin{array}{l}\text { Demokratie } \\
\text { (Index } 0-10)\end{array}$ & 3.9 & 4.1 & 1.8 \\
\hline $\begin{array}{l}\text { Einkommensverteilung } \\
\text { (Gini-Koeffizient) }\end{array}$ & 0.41 & 0.41 & 0.41 \\
\hline $\begin{array}{l}\text { Landverteilung } \\
\text { (Gini-Koeffizient) }\end{array}$ & 0.64 & 0.64 & 0.63 \\
\hline
\end{tabular}

Eine dritte Finanzierungsmöglichkeit sind feindliche Regierungen. Ein Beispiel ist die Finanzierung von Renamo in Mosambik durch die Regierung im damaligen 
Süd-Rhodesien. Unsere Variable für die Bereitschaft ausländischer Regierungen, die militärische Opposition in anderen Ländern zu unterstützen, ist die Periode des Kalten Krieges. Während des Kalten Krieges unterstützten die Supermächte Rebellenorganisationen in Ländern, die mit der anderen Supermacht alliert waren. Bezüglich dieser Finanzquelle finden wir einen schwachen Anhaltspunkt für die Möglichkeits-Theorie: nur elf von 78 Kriegen brachen in den zehn Jahren seit dem Ende des Kalten Krieges aus.

Wir wenden uns nun der Diskussion von Möglichkeiten zu, die aus atypisch niedrigen Kosten entstehen. Rekruten müssen bezahlt werden, und ihre Kosten stehen in Verhältnis zu den entgangenen Einnahmen (Opportunitätskosten) wenn sie sich einer Rebellenorganisation anschliessen. Ein Beispiel, dass diese Opportunitätskosten eine wichtige Determinante sein können, ist der russische Bürgerkrieg. Die Weißrussische und die Rote Armee hatten vier Millionen Deserteure. Im Sommer war die Zahl der desertierten Rebellen viermal so hoch wie im Winter. Die Rebellenarmeen rekrutierten überwiegend von der Landbevölkerung, und deren entgangene Einnahmen waren während der Erntezeit wesentlich höher (Figes, 1996). Desertieren ist das genaue Gegenteil von Rekrutieren und zeigt daher, dass Rekrutierung von den Opportunitätskosten abhängt. Wir benutzen drei Variablen für entgangene Einnahmen: Pro-KopfEinkommen, den Anteil der jungen Männer, die weiterführende Schulen besuchen und die Wachstumsrate des Pro-Kopf-Einkommens. In Tabelle 2 zeigen wir, dass Bürgerkriegsepisoden durch eine wesentlich geringeres Pro-Kopf-Einkommen gekennzeichnet sind, welches in Bürgerkriegsepisoden weniger als die Hälfte des Pro-Kopf Einkommens in Friedensepisoden beträgt. Die zweite Variable, der Anteil der jungen Männer, die weiterführende Schulen besuchen, hat den Vorteil, dass sie sich auf die Gruppe konzentriert, aus der überwiegend rekrutiert wird: junge Männer. Der Anteil der jungen Männer, die weiterführende Schulen besuchen ist wesentlich niedriger in den Bürgerkriegsepisoden. Unsere dritte Variable ist die Wachstumsrate des Pro-Kopf-Einkommens in den fünf vorangegangenen Jahren. Diese Variable gibt uns einen Eindruck über die Einkommenschancen in der Volkswirtschaft. Bürgerkriegsausbrüche sind durch geringes wirtschaftliches Wachstum in den vorangegangenen Jahren gekennzeichnet. Dies stimmt überein mit der empirischen Arbeit von Alesina et al. (1996). Dort wird gezeigt, dass niedrigere Wachstumsraten mit nichtverfassungsgemäßen Regierungswechseln korrelliert sind. Alle drei Variablen sind Näherungsvariablen für Opportunitätskosten, allerdings können niedrige Einkommen auch als objektive ökonomische Leidensfaktoren interpretiert werden.

Ein weiterer wichtiger Kostenfaktor von Rebellionen sind die Kosten von konfliktspezifischem Kapital. Wir fassen unter diesem Begriff sowohl die organisatorischen als auch die Kapitalkosten zusammen. Über längere Zeiträume hinweg verlieren Waffen an Wert und organisatorische Strukturen an Zusammenhalt. Wir messen daher die Kosten von konfliktspezifischem Kapital durch die Anzahl der Friedensmonate seit dem Ende des letzten Konfliktes. 
Empirisch betrachtet sind Bürgerkriegsausbrüche durch wesentlich kürzere vorangegangene Friedensperioden gekennzeichnet. Obwohl dies die MöglichkeitsTheorie zu bestätigen scheint, könnte man diese empirische Evidenz auch als eine Abnahme von kriegsinduziertem Hass über die Zeit hinweg interpretieren.

Eine weitere Dimension von Möglichkeiten ist eine atypisch schlechte Verteidigungsposition der Regierung. Es ist ein unbestreitbarer Vorteil für Rebellen, wenn das Terrain bergig und bewaldet ist, da es gute Rückzugs- und Versteckmöglichkeiten bietet. Wir verwenden Food and Agriculture Organisation (FAO) Daten für den Anteil des Landes, der bewaldet ist. Für den Anteil des Landes, der bergig ist, verwenden wir Daten von Gerrard (2000). Die deskriptiven Statistiken in Tabelle 2 lassen vermuten, dass die Beschaffenheit des Terrains ein wichtiger Faktor in Bürgerkriegen ist. Die Bürgerkriegsepisoden sind durch einen höheren Anteil an Bergen (25 Prozent) gekennzeichnet, dieser Wert beträgt nur etwa 15 Prozent für Friedensepisoden. Im Hinblick auf Bewaldung findet sich praktisch kein Unterschied. Die geographische Verteilung der Bevölkerung mag ebenfalls eine Rolle spielen. In Staaten, in denen die Bevölkerung über das Land verstreut lebt, können Regierungen die Gesamtbevölkerung weniger gut kontrollieren, als bei einem hohen Konzentrationsgrad der Bevölkerung. Herbst (2000) stellt die Hypothese auf, dass Zaire eher zu Konflikten neigt, da die Bevölkerung sehr verstreut an den Grenzen des Landes lebt. Wir stellen die Verteilung der Bevölkerung durch einen Gini-Koeffizienten dar. ${ }^{5}$ Im Gegenteil zu dieser Hypothese zeigen unsere Daten, dass die Konzentration der Bevölkerung in den Friedensepisoden etwas niedriger ist (0.57) als in den Bürgerkriegsepisoden (0.6). Gleichermaßen können eine niedrige Bevölkerungsdichte und ein geringer Urbanisierungsgrad die Effizienz der Regierung verringern. Empirisch betrachtet sind Bürgerkriegsausbrüche häufiger in Ländern zu beobachten, die durch eine niedrige Bevölkerungsdichte und einen niedrigen Urbanisierungsgrad gekennzeichnet sind (siehe Tabelle 2).

Die letzte Dimension der Möglichkeits-Theorie bezieht sich auf soziale Kohäsion. Ethnische und religiöse Diversität reduziert die Funktionstüchtigkeit von Organisationen (Easterly und Levine, 1997, Alesina et al., 1999, Collier, 2001). Um eine möglichst starke Kohäsion innerhalb der Organisation zu erzielen, rekrutieren die Rebellen bevorzugt aus einer ethnischen oder religiösen Gruppe. Eine fragmentierte Gesellschaft bietet daher weniger Möglichkeiten für die Rekrutierung einer großen kohäsiven Rebellenorganisation. Die am häufigsten benutzte Variable für ethnische Diversität ist der Index der ethno-linguistischen Fraktionalisierung. ${ }^{6}$ Dieser Index misst die Wahrscheinlichkeit, dass zwei zufällig

5 Für die Kalkulation des Bevölkerungs-Gini-Koeffizienten benutzen wir die Bevölkerung pro $400 \mathrm{~km}^{2}$ Zellen. Analog zum Einkommens-Gini-Koeffizienten ist der Bevölkerung-Gini-Koeffizient hoch, wenn die Bevölkerung stark in einem Teil des Landes konzentriert ist.

6 Mauro (1995) verwendete diesen Index als erster in der ökonomischen Literatur. 
herausgegriffene Menschen nicht der gleichen ethno-linguistischen Gruppe angehören. Ein Wert von Null indiziert daher eine vollständig homogene Gesellschaften, während höhere Werte einen höheren Grad an Diversität implizieren. Da wir keinen vergleichbaren Index für religiöse Diversität finden konnten, verwenden wir die Daten aus Barrett (1982), um einen äquivalenten Index zu erhalten. Wenn ethnische und religiöse Unterschiede nicht die gleichen Gruppen beschreiben, sondern diese sich überschneiden, ist soziale Fragmentierung ein Produkt der beiden Variablen und nicht deren Summe. Wir konnten keine Informationen über den Überschneidungsgrad ethnischer und religöser Gruppen finden und nehmen daher das Produkt der beiden Variablen als maximal mögliche soziale Fragmentierung. ${ }^{7}$ Wie Tabelle 2 zeigt, sind Konfliktepisoden durch stärkere soziale Fragmentierung gekennzeichnet. Die Hypothese, dass soziale Kohäsion das Konfliktrisiko verringert, wird durch die deskriptiven Statistiken unterstützt: Bürgerkriegsländer haben eine ungewöhnlich hohe ethnische Fragmentierung. Dies scheint konsistent mit der Leidens-Theorie zu sein, die wir nun genauer erläutern möchten.

\section{Objektive Messwerte für Leid}

Wie bereits diskutiert, ist Leid ein universelles Phänomen. Es ist relativ schwierig, objektive Messwerte für die Stärke des Leidens zu finden. Wir benutzen die folgenden vier Konzepte: ethnischer und religiöser Hass, politische Repression, politischer Ausschluss und ökonomische Ungleichheit.

Oft werden ethnischer und religöser Hass als die Ursache von Bürgerkriegen angeführt. Obwohl dieser Hass nicht quantifiziert werden kann, kann er nur in ethnisch und religiös fragmentierten Gesellschaften vorkommen. Die oben besprochenen Variablen ethnischer und religiöser Fragmentierung können auch als relevante Messwerte für Inter-Gruppen-Hass interpretiert werden. Je höher die Anzahl der unterschiedlichen Gruppen, desto größer ist der mögliche Hass zwischen diesen. Allerdings kann man auch argumentieren, dass dem InterGruppen-Hass nicht Diversität, sondern Polarisierung zugrunde liegt. Diese Hypothese wird z.B. von Esteban und Ray (1999) und von Reynal-Querol (2001) vertreten. Wir verwenden das Konzept von Esteban und Ray (1994), um die ethnische Polarisierung zu erfassen. ${ }^{8}$ Die deskriptiven Statistiken veranlassen uns allerdings nicht $\mathrm{zu}$ der Annahme, dass Polarisierung mit einem höheren

$7 \quad$ Im Fall von $e$ gleich grossen ethnischen Gruppen und $r$ gleich grossen religiösen Gruppen, wird die maximale soziale Fragmentierung durch das Produkt $e \cdot r$ gemessen. Da einer oder beide Indizes null sein kann, erfassen wir soziale Fragmentierung durch das Produkt und addieren den größeren der beiden Indizes.

8 Wir folgen hier der Anwendung von Reynol-Querol (2001). Eine detaillierte Beschreibung ist in Collier und Hoeffler (2001) dargestellt. 
Bürgerkriegsrisiko verbunden ist, da Bürgerkriegsepisoden einen ähnlichen Mittelwert wie Friedensepisoden aufweisen.

Politische Unterdrückung ist eine weitere Leidensvariable, die wir mit Hilfe der Variablen des Polity III Datensatzes erfassen (siehe Jaggers und Gurr, 1995). Die Variable "Demokratie" misst auf einer Skala von 0 bis 10 die Offenheit von politischen Systemen, wobei höhere Werte mit einem höheren Grad an Offenheit assoziiert sind. Wie Tabelle 2 zeigt, unterscheiden sich die Bürgerkriegsepisoden in erheblichem Maße von den Friedensepisoden. Der Mittelwert der Demokratievariablen, die wir am Anfang jeder fünf Jahresperiode erfassen, beträgt für Bürgerkriegsepisoden weniger als die Hälfte des Mittelwertes für Friedensepisoden. ${ }^{9}$ Die quantitative Literatur der Politikwissenschaften hat diese Variablen bereits in Panel-Konfliktstudien untersucht. Hegre et al. (2001) fanden, dass politische Unterdrückung das Bürgerkriegsrisiko erhöht, allerdings mit der Einschränkung, dass sehr starke Repression Bürgerkriege unterdrückt.

Selbst in demokratischen Systemen können Minderheiten unterdrückt werden, z.B. wenn politische Zugehörigkeit durch ethnische Gruppen bestimmt wird. Eine ethnische Gruppe, welche die Mehrheit stellt, kann die Minderheit ausbeuten. Diese Form der Ausbeutung ist allerdings nur dann lukrativ, wenn die Minderheit eine relativ große Gruppe darstellt. Wir nennen dies "ethnische Dominanz" und definieren diesen Zustand, wenn die größte ethnische Gruppe zwischen 45 und 90 Prozent der Gesamtbevölkerung stellt. Wie Tabelle 2 zeigt, scheint dieses Phänomen nicht wichtig zu sein, da Bürgerkriegsepisoden ebenso häufig wie Friedensepisoden durch ethnische Dominanz gekennzeichnet sind.

Ungleichheit wird ebenfalls häufig als Ursache von Bürgerkriegen angegeben. Auf der ersten Seite von Sens "On Economic Inequality" findet sich die Hypothese, dass "die Verbindung von Ungleichheit und Rebellionen eng ist". Die Motivation der Armen zur Rebellion kann Umverteilung sein, andererseits können reiche Regionen durch einen Sezessionskrieg versuchen, Umverteilung zu verhindern. ${ }^{10}$ Wir erfassen Ungleichheit mit dem Gini-Koeffizienten der Einkommensverteilung und dem Quotienten des Einkommensanteils des obersten zum untersten Fünftel. Vermögensungleichheit erfassen wir mit dem Gini-Koeffizienten der Landverteilung. Alle Verteilungsdaten wurden von Deininger und Squire (1996, 1998) zur Verfügung gestellt. Es zeigt sich, dass die Verteilung von Einkommen und Vermögen etwas ungleicher in den Bürgerkriegs- als in den Friedensepisoden ist.

9 Wir untersuchten auch die Variable "Autokratie" des gleichen Datensatzes, sowie die häufig verwandte Variable "Politische Rechte", Datenquelle: http://www.freedomhouse.org:80/index.htm.

10 Dies ist eine Parallele zur Theorie des Steueraustrittes, beschrieben in Buchanan und Faith (1987). 


\section{Skaleneffekte}

Der Effekt der Größe des Landes auf das Bürgerkriegsrisiko kann uns möglicherweise helfen, zwischen der Möglichkeits- und der Leidens-Theorie zu unterscheiden. Es ist plausibel anzunehmen, dass Möglichkeiten, wie natürliche Ressourcen oder charismatische Rebellenführer, ungefähr proportional mit der Größe des Landes zunehmen. Es ist allerdings unwahrscheinlich, dass objektive Leidensfaktoren, wie z.B. politische Unterdrückung mit der Größe der Gesamtbevölkerung verbunden sind. Empirisch betrachtet haben Bürgerkriegsländer eine wesentlich größere Bevölkerung als friedliche Länder.

\section{Empirische Analyse}

In unserer empirischen Arbeit analysieren wir das Risiko eines Kriegsausbruches in fünf-Jahres Perioden, wofür wir Logit Regressionen anwenden. Unser Panel besteht aus 161 Ländern und acht Perioden, 1960-64, 1965-69 ... 1995-99. Die abhängige Variable nimmt den Wert null an, wenn es in dem Land während der gesamten fünf Jahre friedlich war und einen Wert von eins, wenn ein Bürgerkrieg ausbrach. Wir betrachten nur das Ereignis des Bürgerkriegsausbruches, nicht aber die Dauer des Krieges. ${ }^{11}$ Für einige Variablen haben wir keine Zeitreihendaten und verwenden daher die gleichen Beobachtungen für alle Perioden. Die Koeffizienten der Variablen geben an, ob Länder mit bestimmten Charakteristika ein deutlich höheres oder niedrigeres Risiko eines Bürgerkriegsbruches haben.

Wir beginnen mit der Analyse des Möglichkeiten-Modells (siehe Tabelle 3). Da das Pro-Kopf-Einkommen und der Anteil junger Männer, die weiterführende Schulen besuchen, stark miteinander korreliert sind, verwenden wir diese beiden erklärenden Variablen nicht in der gleichen Modellspezifikation. Die Spezifikation in der ersten Spalten verwendet den Anteil junger Männer, die weiterführende Schulen besuchen, aber nicht das Pro-Kopf-Einkommen.

11 Die Determinanten der Kriegsdauer werden in Collier, Hoeffler und Söderbom (2001) untersucht. Ihre Ergebnisse zeigen, dass der Ausbruch und die Dauer von Bürgerkriegen nicht von den gleichen Faktoren bestimmt werden. In einer früheren Arbeit (Collier und Hoeffler, 1998) unterschieden wir nicht deutlich zwischen Ausbruch und Dauer und benutzten Tobit Regressionen. 
Tabelle 3: Möglichkeiten-Modell

\begin{tabular}{|c|c|c|c|c|}
\hline & \multicolumn{4}{|c|}{$\begin{array}{l}\text { Logit Regressionen } \\
\text { Ereignis: Bürgerkriegsausbrüche }\end{array}$} \\
\hline & 1 & 2 & 3 & 4 \\
\hline Schulbesuch junger Männer & $\begin{array}{l}-0.024 \\
(0.010)^{* *}\end{array}$ & & & \\
\hline ln Pro-Kopf Einkommen & & $\begin{array}{l}-0.837 \\
(0.253) * * *\end{array}$ & $\begin{array}{l}-1.237 \\
(0.283)^{* * * *}\end{array}$ & $\begin{array}{l}-1.243 \\
(0.284) * * *\end{array}$ \\
\hline Einkommenswachstum & $\begin{array}{l}-0.118 \\
(0.044)^{* * *}\end{array}$ & $\begin{array}{l}-0.105 \\
(0.042)^{* * *}\end{array}$ & & \\
\hline Primärproduktexporte/BSP & $\begin{array}{l}18.900 \\
(5.948)^{* * *}\end{array}$ & $\begin{array}{l}16.476 \\
(5.207)^{* * *}\end{array}$ & $\begin{array}{l}17.567 \\
(6.744)^{* * * *}\end{array}$ & $\begin{array}{l}17.404 \\
(6.750)^{* * *}\end{array}$ \\
\hline (Primärproduktexporte/BSP) $^{2}$ & $\begin{array}{l}-29.123 \\
(11.905)^{* * *}\end{array}$ & $\begin{array}{l}-23.017 \\
(9.972) * *\end{array}$ & $\begin{array}{l}-28.815 \\
(15.351)^{*}\end{array}$ & $\begin{array}{l}-28.456 \\
(15.366)^{*}\end{array}$ \\
\hline Soziale Fragmentierung & $\begin{array}{l}-0.0002 \\
(0.0001) \\
\mathrm{p}=0.122 \\
\end{array}$ & $\begin{array}{l}-0.0002 \\
(0.0001)^{* *}\end{array}$ & & \\
\hline Friedensperiode & $\begin{array}{l}-0.004 \\
(0.001) * * *\end{array}$ & $\begin{array}{l}-0.004 \\
(0.001)^{* * * *}\end{array}$ & $\begin{array}{l}-0.002 \\
(0.001)\end{array}$ & $\begin{array}{l}-0.002 \\
(0.001)\end{array}$ \\
\hline Post Kalter Krieg & $\begin{array}{l}-0.207 \\
(0.450)\end{array}$ & $\begin{array}{l}-0.454 \\
(0.416) \\
\end{array}$ & & \\
\hline $\begin{array}{l}\text { Diaspora/ } \\
\text { Friedensperiode }\end{array}$ & & & $\begin{array}{l}700.931 \\
(363.29)^{* *}\end{array}$ & \\
\hline $\begin{array}{l}\text { Diaspora korrigiert/ } \\
\text { Friedensperiode }\end{array}$ & & & & $\begin{array}{l}741.168 \\
(387.635)^{*}\end{array}$ \\
\hline $\begin{array}{l}\text { (Diaspora-Diaspora korrigiert)/ } \\
\text { Friedensperiode }\end{array}$ & & & & $\begin{array}{l}82.798 \\
(287.192) \\
\end{array}$ \\
\hline ln Bevölkerung & $\begin{array}{l}0.686 \\
(0.162)^{* * *}\end{array}$ & $\begin{array}{l}0.493 \\
(0.129) * * *\end{array}$ & $\begin{array}{l}0.295 \\
(0.141)^{* *}\end{array}$ & $\begin{array}{l}0.296 \\
(0.141)^{* *}\end{array}$ \\
\hline Verteilung der Bevölkerung & $\begin{array}{l}-2.129 \\
(1.032) * *\end{array}$ & $\begin{array}{l}-0.865 \\
(0.948)\end{array}$ & & \\
\hline Bergiges Terrain & $\begin{array}{l}0.014 \\
(0.009)\end{array}$ & $\begin{array}{l}0.008 \\
(0.008)\end{array}$ & & \\
\hline $\mathrm{N}$ & 688 & 750 & 595 & 595 \\
\hline $\begin{array}{l}\text { Anzahl der } \\
\text { Bürgerkriegsausbrüche }\end{array}$ & 46 & 52 & 29 & 29 \\
\hline Pseudo $\mathrm{R}^{2}$ & 0.24 & 0.22 & 0.25 & 0.25 \\
\hline Log likelihood & -128.85 & -146.86 & -93.27 & -93.23 \\
\hline
\end{tabular}

Bemerkungen: Alle Regressionen haben eine Konstante. Standardfehler in Klammern, p indiziert das Signifikanzniveau. ***, **, * indizieren Signifikanz auf dem 1, 5 and 10 Prozent-Niveau.

Für diese Schätzung können wir 688 Beobachtungen (122 Länder) verwenden, von denen 46 durch einen Kriegsausbruch charakterisiert sind. In der zweiten Spalte untersuchen wir eine ähnliche Spezifikation, allerdings benutzen wir hier das pro-Kopf Einkommen. In dieser Modellspezifikation können wir 750 Beobachtungen (125 Länder) verwenden, von denen 52 durch den Ausbruch eines Bürgerkrieges gekennzeichnet sind.

In der Schätzung, die in der ersten Spalte aufgeführt ist, haben die beiden Variablen, die die Opportunitätskosten messen, die erwarteten Vorzeichen und 
sind statistisch signifikant. Ein hoher Anteil junger Männer, die weiterführende Schulen besuchen, und ein hohes Einkommenswachstum in den vorangegangenen fünf Jahren reduzieren das Bürgerkriegsrisiko. Der Effekt von natürlichen Ressourcen auf das Risiko eines Bürgerkriegsausbruches ist ebenfalls signifikant, allerdings ist dieser nicht monoton. Das Risiko eines Bürgerkrieges ist am höchsten, wenn der Anteil der Primärproduktexporte ungefähr $32 \%$ des Bruttosozialproduktes ausmacht. Der positive Effekt ist konsistent mit der Hypothese, dass Primärproduktexporte eine Finanzquelle darstellen. Ein höherer Anteil von Primärproduktexporten erhöht daher das Bürgerkriegsrisiko. Der negative quadratische Effekt kann damit erklärt werden, dass primäre Rohstoffe nicht nur eine Finanzierungsquelle für die Rebellen darstellen, sondern auch für die Regierung. In vielen Entwicklungsländern stellt die Besteuerung von Rohstoffen einen großen Teil der Staatseinnahmen dar. Die Regierung eines Landes mit hohem Primärproduktanteil am Bruttosozialprodukt hat daher die Möglichkeit, mehr für Verteidigung auszugeben und potentielle Rebellionen im Ansatz zu verhindern. Aus diesem Grund reduziert ein sehr hoher Primärproduktanteil das Bürgerkriegsrisiko. Die Signifikanz einer weiteren möglichen Finanzierungsquelle, die beiden Supermächte während des Kalten Krieges, testen wir, indem wir eine Dummy-Variable für die Perioden seit dem Ende des Kalten Krieges im Modell einfügen (1990-94 und 1995-99). Das Vorzeichen ist zwar wie erwartet negativ, die Variable ist allerdings nicht statistisch signifikant.

Der Koeffizient der Friedensperiode seit dem Ende des letzten Bürgerkrieges ist negativ, d.h. mit der Länge der Friedensperiode nimmt das Risiko eines erneuten Krieges ab. Soziale Fragmentierung ist wie erwartet negativ, die Variable ist allerdings marginal insignifikant in dieser Spezifikation. Der Koeffizient für bergiges Terrain hat ein positives Vorzeichen, ist aber nicht signifikant. Eine höhere Konzentration der Bevölkerung reduziert das Bürgerkriegsrisiko und die Elastizität des Bürgerkriegsrisikos im Hinblick auf die Gesamtbevölkerung ist kleiner als eins.

Soweit zeigen unsere Ergebnisse, dass Möglichkeiten helfen, Bürgerkriegsausbrüche zu erklären. Während des Krieges wird konfliktspezifisches Kapital gebildet wird, das dann weitere Konflikte in der Zukunft verursachen kann. In der zweiten Spalte führen wir eine alternative Spezifikation an, in der wir die Opportunitätskosten mit dem Pro-Kop-Einkommen anstelle des Anteils junger Männer, die weiterführende Schulen besuchen erfassen. Die Ergebnisse sind sehr ähnlich, allerdings erklärt dieses Modell das Kriegsrisiko nicht ganz so gut wie unser Modell in Spalte 1. Der Koeffizient der sozialen Fragmentierung ist nun signifikant, d.h. fragmentierte Gesellschaften haben ein geringeres Konfliktrisiko.

Die Länge der Friedensperiode wurde von uns bislang als kriegsspezifisches Kapital interpretiert, allerdings könnte man sie auch als Indikator für Hass ansehen. Wir versuchen nun zwischen diesen beiden Erklärungsversuchen zu 
unterscheiden, indem wir den Zusammenhang zwischen der Friedensperiode und der Größe der Diaspora analysieren. Da wir relativ wenige Daten über die Größe der Diaspora in den USA haben, verringert sich unsere Stichprobe. Um eine möglichst große Stichprobe verwenden zu können, reduzieren wir unser Modell, indem wir vier weniger wichtige Determinanten vernachlässigen: ethnische und religiöse Fragmentierung, die geographische Konzentration der Bevölkerung, der Anteil des Landes, der bergig ist und das Pro-Kopf-Wirtschaftswachstum der letzten fünf Jahre. Selbst in diesem stark reduzierten Modell können wir nur 595 Beobachtungen mit 32 Kriegen untersuchen. Um den sich abschwächenden postKonflikt Effekt zu berücksichtigen, lassen wir unsere Diaspora-Variable mit der Anzahl der Friedensmonate seit dem letzten Bürgerkrieg interagieren, indem wir ihren Quotienten bilden (Diaspora/Friedensepisode). Der Koeffizient ist positiv und signifikant, allerdings verlieren die Friedensmonate an statistischer Signifikanz ( $\mathrm{p}=0.14$ ). Eine größere Diaspora vergrößert das Risiko eines neuen Bürgerkrieges erheblich: post-Konflikt-Länder mit der größten Diaspora haben ein sechsfach höheres Risiko als Länder mit der kleinsten Diaspora. ${ }^{12}$

Obwohl dies die These unterstützt, dass Diasporen Konflikte finanzieren, kann man diese Ergebnisse auch anders interpretieren. Diasporen können auch im Hinblick auf die Intensität der Bürgerkriege endogen sein. Wenn ein Bürgerkrieg ausbricht, fliehen viele Menschen, ein Teil wandert in die USA aus, d.h. die Diaspora ist vielleicht nur ein Merkmal der Intensität des Konfliktes. In diesem Fall können unsere Ergebnisse so interpretiert werden, dass Länder mit besonders schweren Konflikten ein erhöhtes Risiko für neue Bürgerkriege haben. Um diese unterschiedlichen Theorien zu testen, zerlegen wir die Diaspora in zwei Komponenten, eine exogene und eine endogene. Um den exogenen Teil zu bestimmen, schätzen wir ein einfaches Migrationsmodell. ${ }^{13}$ Für die Beobachtungen nach einem Bürgerkrieg substituieren wir die tatsächliche mit der geschätzten Größe der Diaspora. Auf diese Art "bereinigen" wir die DiasporaVariable von dem Anteil der durch die Intensität der vorangegangenen Bürgerkriege bestimmt wurde. Die Differenz der tatsächlichen Diaspora und dieses "bereinigten" Wertes kann dann als endogene Diaspora interpretiert werden. Beide Variablen werden nun anstelle der tatsächlichen Diaspora in der letzten Spalte von Tabelle 3 eingeführt. Der "bereinigte" Wert der Diaspora ist signifikant, die Größe des Koeffizienten unterscheidet sich nur geringfügig von dem der tatsächlichen Diaspora in der vorangegangenen Regressionsgleichung. Der Koeffizient der endogenen Diaspora Variablen hingegen ist nicht signifikant. Wenn die Diaspora ein Merkmal der Intensität des Konfliktes wäre, wäre zu

12 Diese Berechnung basiert auf der Annahme, dass alle anderen Variablen den Mittelwert annehmen und die post-Konflikt Friedensperiode fünf Jahre ist.

13 Die Größe der Diaspora wird als eine Funktion der Diaspora, des pro-KopfEinkommens und einer Bürgerkriegs-Dummy in der vorangegangenen Periode beschrieben (die Ergebnisse sind in Hoeffler und Collier (2001) dargestellt). 
erwarten, dass der Koeffizient der "bereinigten" Variablen insignifikant und der Koeffizient der endogenen Diaspora signifikant ist.

Diese Ergebnisse lassen die Schlussfolgerung zu, dass ein kausaler Zusammenhang zwischen der Diaspora und dem Bürgerkriegsrisiko besteht, der sich am besten durch die finanzielle Unterstützung der Rebellen durch die Diaspora erklären lässt. Dies ist nicht nur im Hinblick auf die Rolle der Diasporen interessant, sondern sie helfen den Effekt der Friedensperiode auf das Bürgerkriegsrisiko zu interpretieren. Im vorangegangenen Abschnitt schlugen wir zwei mögliche Wirkungen der Länge der Friedensperiode auf das Bürgerkriegsrisiko vor. Einerseits verliert das kriegsspezifischen Kapital an Wert, andererseits schwächt sich der Hass über die Zeit hinweg ab. Wenn der Friedenseffekt durch das kriegsspezifische Kapital wirkt, ist es wahrscheinlich, dass die Diaspora durch finanzielle Zuwendungen diese Abdiskontierung des Kapitals verhindert oder sogar neue Investitionen zulässt. Wenn der Hass-Effekt überwiegen würde, wäre anzunehmen, dass eine größere Diaspora einen verstärkten Hass im Heimatland schürt. Da die Diaspora aber im Vergleich zur Bevölkerung im Heimatland sehr klein ist und Kommunikationsmöglichkeiten begrenzt sind, halten wir diese Erklärung für unwahrscheinlich. Wir erklären den Diaspora-Friedenseffekt durch den überproportionalen finanziellen Einfluss der Diaspora auf die Bevölkerung im Heimatland und nicht durch einen überproportionalen Einfluß auf deren Einstellung. 
Tabelle 4: Leid-Modell

\begin{tabular}{|c|c|c|c|}
\hline & \multicolumn{3}{|c|}{$\begin{array}{l}\text { Logit Regressionen, Ereignis: } \\
\text { Bürgerkriegsausbrüche }\end{array}$} \\
\hline & 1 & 2 & 3 \\
\hline Ethnische Fragmentierung & $\begin{array}{l}0.010 \\
(0.006)^{*}\end{array}$ & $\begin{array}{l}0.011 \\
(0.007)^{*}\end{array}$ & $\begin{array}{l}0.012 \\
(0.008) \\
\end{array}$ \\
\hline Religiöse Fragmentierung & $\begin{array}{l}-0.003 \\
(0.007)\end{array}$ & $\begin{array}{l}-0.006 \\
(0.008)\end{array}$ & $\begin{array}{l}-0.004 \\
(0.009)\end{array}$ \\
\hline Polarisation $\alpha=1.6$ & $\begin{array}{l}-3.067 \\
(7.021)\end{array}$ & $\begin{array}{l}-4.682 \\
(8.267)\end{array}$ & $\begin{array}{l}-6.536 \\
(8.579)\end{array}$ \\
\hline Ethnische Dominanz (45-90\%) & $\begin{array}{l}0.414 \\
(0.496)\end{array}$ & $\begin{array}{l}0.575 \\
(0.586) \\
\end{array}$ & $\begin{array}{l}1.084 \\
(0.629)^{*}\end{array}$ \\
\hline Demokratie & $\begin{array}{l}-0.109 \\
(0.044) * * *\end{array}$ & $\begin{array}{l}-0.083 \\
(0.051)^{*}\end{array}$ & $\begin{array}{l}-0.121 \\
(0.053)^{* *}\end{array}$ \\
\hline Friedensperiode & $\begin{array}{l}-0.004 \\
(0.001)^{* * *}\end{array}$ & $\begin{array}{l}-0.003 \\
(0.001)^{* * *}\end{array}$ & $\begin{array}{l}-0.004 \\
(0.001)^{* * *}\end{array}$ \\
\hline Einkommensverteilung & & $\begin{array}{l}0.015 \\
(0.018) \\
\end{array}$ & \\
\hline Landverteilung & & & $\begin{array}{l}0.461 \\
(1.305)\end{array}$ \\
\hline ln Bevölkerung & $\begin{array}{l}0.221 \\
(0.096) * *\end{array}$ & $\begin{array}{l}0.246 \\
(0.119)^{* *}\end{array}$ & $\begin{array}{l}0.300 \\
(1.133)^{* *}\end{array}$ \\
\hline Verteilung der Bevölkerung & $\begin{array}{l}-0.509 \\
(0.856)\end{array}$ & $\begin{array}{l}-0.763 \\
(1.053)\end{array}$ & $\begin{array}{l}-1.293 \\
(0.102)\end{array}$ \\
\hline Bergiges Terrain & $\begin{array}{l}0.011 \\
(0.007)\end{array}$ & $\begin{array}{l}0.007 \\
(0.009)\end{array}$ & $\begin{array}{l}-0.0001 \\
(0.009)\end{array}$ \\
\hline $\mathrm{N}$ & 850 & 604 & 603 \\
\hline $\begin{array}{l}\text { Anzahl der } \\
\text { Bürgerkriegsausbrüche }\end{array}$ & 59 & 41 & 38 \\
\hline Pseudo $\mathrm{R}^{2}$ & 0.13 & 0.11 & 0.17 \\
\hline Log likelihood & -185.57 & -133.46 & -117.12 \\
\hline
\end{tabular}

Bemerkungen: Alle Regressionen haben eine Konstante. Standardfehler in Klammern. ***, $* *, *$ indizieren Signifikanz auf dem 1, 5 and 10 Prozent-Niveau.

Im Modell in Tabelle 4 testen wir die Hypothese, ob Bürgerkriege durch objektiv messbare Leidensfaktoren verursacht werden. In diesem Modell vernachlässigen wir alle Approximationen der ökonomischen Möglichkeiten, aber wir verwenden die Länge der Friedensperiode, da diese möglicherweise den sich abschwächenden Hass abbildet. Im Modell der ersten Spalte berücksichtigen wir den Effekt von Ungleichheit nicht, da sich durch die vielen fehlenden Beobachtungen unsere Stichprobe stark reduzieren würde. Mit dieser Modellspezifikation können wir 850 Beobachtungen mit 59 Bürgerkriegsepisoden analysieren.

Die vier Variablen, die objektiv messbare Leidensfaktoren abbilden, sind relativ unwichtig, und es ist daher erstaunlich, wieviel Beachtung sie in der Diskussion um die Erklärung von Bürgerkriegen erfahren. Ethnische Fragmentierung ist signifikant (auf einem Signifikanzniveau von 10 Prozent) und hat das erwartete 
positive Vorzeichen. Religiöse Fragmentierung, ethnische Polarisierung und Dominanz sind sowohl einzeln als auch gemeinsam nicht signifikant. Demokratie ist hochsignifikant mit dem erwarteten Vorzeichen, politische Unterdrückung erhöht das Bürgerkriegsrisiko. Die Friedensperiode ist ebenfalls signifikant, allerdings ist es möglich, dass diese Variable, wie bereits erwähnt, keinen objektiven Leidensfaktor darstellt. In der zweiten und dritten Spalte testen wir die Hypothese, ob Ungleichheit das Bürgerkriegsrisiko erhöht. Unsere Stichprobe wird auf etwa 600 Beobachtungen verringert und weder Einkommens- noch Landverteilung sind statistisch signifikant (Tabelle 4: Spalten 3 und 4). Alle drei Leid-Modelle haben einen geringen Erklärungswert, die Regressionen haben einen $\mathrm{R}^{2}$-Wert von maximal 0.17.

Wir untersuchen nun, welches Modell, das Möglichkeiten- oder das Leid-Modell, eine bessere Erklärung für das Bürgerkriegsrisiko bietet. Da die beiden Modelle unterschiedlich und nicht ineinander überführbar sind (non-nested), benutzen wir den J-Test von Davidson und McKinnon (1981) um diese Frage zu beantworten. Aufgrund dieses Testes können wir keines der beiden Modelle zugunsten des anderen ablehnen. ${ }^{14}$ Wir stellen somit fest, dass einige Leidfaktoren das Möglichkeiten-Modell verbessern, obwohl es insgesamt eine bessere Erklärung des Bürgerkriegsrisikos als das Leid-Modell bietet. Wir untersuchen daher eine Kombination der beiden Modelle in Spalte 1 in Tabelle 5.

14 Die Ergebnisse des J-Tests stellen wir in Collier und Hoeffler (2001) dar. 
Tabelle 5: Kombiniertes Möglichkeiten- und Leid-Modell

\begin{tabular}{|c|c|c|c|c|}
\hline & \multicolumn{4}{|c|}{ Logit Regressionen, Ereignis: Bürgerkriegsausbrüche } \\
\hline & 1 & 2 & 3 & 4 \\
\hline $\begin{array}{l}\text { Schulbesuch junger } \\
\text { Männer }\end{array}$ & $\begin{array}{l}-0.029 \\
(0.013)^{* *}\end{array}$ & $\begin{array}{l}-0.022 \\
(0.011)^{* *}\end{array}$ & $\begin{array}{l}-0.031 \\
(0.010)^{* * *}\end{array}$ & \\
\hline $\begin{array}{l}\ln \text { Pro-Kopf } \\
\text { Einkommen }\end{array}$ & & & & $\begin{array}{l}-0.950 \\
(0.245)^{* * * *}\end{array}$ \\
\hline $\begin{array}{l}\text { Einkommens- } \\
\text { wachstum }\end{array}$ & $\begin{array}{l}-0.045 \\
(0.062)\end{array}$ & $\begin{array}{l}-0.108 \\
(0.045)^{* *}\end{array}$ & $\begin{array}{l}-0.115 \\
(0.043)^{* * *}\end{array}$ & $\begin{array}{l}-0.098 \\
(0.042)^{* *}\end{array}$ \\
\hline $\begin{array}{l}\text { Primärproduktexp./ } \\
\text { BSP }\end{array}$ & $\begin{array}{l}37.072 \\
(10.293)^{* * * *}\end{array}$ & $\begin{array}{l}23.385 \\
(6.692)^{* * * *}\end{array}$ & $\begin{array}{l}18.937 \\
(5.865) * * *\end{array}$ & $\begin{array}{l}16.773 \\
(5.206) * * *\end{array}$ \\
\hline $\begin{array}{l}\text { (Primärproduktexp./ } \\
\mathrm{BSP})^{2}\end{array}$ & $\begin{array}{l}-69.267 \\
(21.697)^{* * * *}\end{array}$ & $\begin{array}{l}-36.335 \\
(12.998)^{* * *}\end{array}$ & $\begin{array}{l}-29.443 \\
(11.781)^{* * * *}\end{array}$ & $\begin{array}{l}-23.800 \\
(10.040)^{* *}\end{array}$ \\
\hline $\begin{array}{l}\text { Soziale } \\
\text { Fragmentierung }\end{array}$ & $\begin{array}{l}-0.0008 \\
(0.0003)^{* *}\end{array}$ & $\begin{array}{l}-0.0005 \\
(0.0003) \mathrm{p}=0.11\end{array}$ & $\begin{array}{l}-0.0002 \\
(0.0001)^{* * *}\end{array}$ & $\begin{array}{l}-0.0002 \\
(0.0001)^{* * * *}\end{array}$ \\
\hline $\begin{array}{l}\text { Ethnische } \\
\text { Fragmentierung }\end{array}$ & $\begin{array}{l}0.041 \\
(0.019)^{* *}\end{array}$ & $\begin{array}{l}0.023 \\
(0.015) \\
\end{array}$ & & \\
\hline $\begin{array}{l}\text { Religiöse } \\
\text { Fragmentierung }\end{array}$ & $\begin{array}{l}0.015 \\
(0.020) \\
\end{array}$ & $\begin{array}{l}0.014 \\
(0.019)\end{array}$ & & \\
\hline Polarisation & $\begin{array}{l}-25.276 \\
(13.390)^{*}\end{array}$ & $\begin{array}{l}-15.992 \\
(10.518)\end{array}$ & & \\
\hline $\begin{array}{l}\text { Ethn. Dominanz } \\
(45-90 \%)\end{array}$ & $\begin{array}{l}2.020 \\
(0.915)^{* *}\end{array}$ & $\begin{array}{l}1.592 \\
(0.746)^{* *}\end{array}$ & $\begin{array}{l}0.670 \\
(0.354)^{*}\end{array}$ & $\begin{array}{l}0.480 \\
(0.328) p=0.14\end{array}$ \\
\hline Demokratie & $\begin{array}{l}-0.018 \\
(0.062)\end{array}$ & $\begin{array}{l}-0.042 \\
(0.054)\end{array}$ & & \\
\hline Friedensperiode & $\begin{array}{l}-0.0003 \\
(0.0015) \\
\end{array}$ & $\begin{array}{l}-0.003 \\
(0.001)^{* * * *}\end{array}$ & $\begin{array}{l}-0.004 \\
(0.001)^{* * *}\end{array}$ & $\begin{array}{l}-0.004 \\
(0.001)^{* * *}\end{array}$ \\
\hline Post Kalter Krieg & $\begin{array}{l}-0.873 \\
(0.644) \\
\end{array}$ & $\begin{array}{l}-0.281 \\
(0.459) \\
\end{array}$ & & \\
\hline $\begin{array}{l}\text { Einkommens- } \\
\text { verteilung }\end{array}$ & $\begin{array}{l}0.025 \\
(0.024)\end{array}$ & & & \\
\hline $\ln$ Bevölkerung & $\begin{array}{l}0.927 \\
(0.250)^{* * * *}\end{array}$ & $\begin{array}{l}0.697 \\
(0.181)^{* * *}\end{array}$ & $\begin{array}{l}0.768 \\
(0.166)^{* * * *}\end{array}$ & $\begin{array}{l}0.510 \\
(0.128)^{* * * *}\end{array}$ \\
\hline $\begin{array}{l}\text { Verteilung der } \\
\text { Bevölkerung }\end{array}$ & $\begin{array}{l}-4.032 \\
(1.490) * * *\end{array}$ & $\begin{array}{l}-1.962 \\
(1.149)^{*}\end{array}$ & $\begin{array}{l}-2.487 \\
(1.005)^{* *}\end{array}$ & $\begin{array}{l}-0.992 \\
(0.909)\end{array}$ \\
\hline Bergiges Terrain & $\begin{array}{l}0.005 \\
(0.012)\end{array}$ & $\begin{array}{l}0.015 \\
(0.009) \mathrm{p}=0.11\end{array}$ & & \\
\hline $\mathrm{N}$ & 479 & 665 & 688 & 750 \\
\hline $\begin{array}{l}\text { Anzahl der Bürger- } \\
\text { kriegsausbrüche }\end{array}$ & 32 & 46 & 46 & 52 \\
\hline Pseudo $\mathrm{R}^{2}$ & 0.24 & 0.26 & 0.24 & 0.22 \\
\hline Log likelihood & -89.55 & -124.60 & -128.21 & -146.84 \\
\hline
\end{tabular}

Bemerkungen: Alle Regressionen haben eine Konstante. Standardfehler in Klammern, p indiziert das Signifikanzniveau. ***,**,* indizieren Signifikanz auf dem 1, 5 and 10 Prozent-Niveau.

Da dieses Modell die Variable Einkommensverteilung beinhaltet, ist unsere Stichprobe stark reduziert (479 Beobachtungen). Da Ungleichheit in dieser und in allen anderen Regressionen insignifikant ist, vernachlässigen wir die Variable im darauffolgenden Modell. In dieser Modellspezifikation können wir 665 
Beobachtungen untersuchen. Weder Demokratie, ethnische und religiöse Diversität noch der Faktor des Endes des Kalten Krieges sind in diesem Kombinationsmodell signifikant. Die anderen Variablen sind signifikant auf dem 10 Prozent-Niveau (oder nahezu) und der Gesamterklärungswert ist relativ hoch $\left(\mathrm{R}^{2}=0.26\right)$. Da nun beide Modelle, das Möglichkeiten- und das Leid-Modell, Teilmodelle dieser Spezifikation sind, können wir sie gegeneinander testen. Wir benutzen Likelihood Ratio Tests und können die Restriktionen des Leid-Modelles ablehnen, aber nicht die Restriktionen des Möglichkeiten-Modelles. ${ }^{15}$

Obwohl das Kombinationsmodell sowohl dem Möglichkeiten- als auch dem LeidModell überlegen ist, sind einige Variablen insignifikant und wir eliminieren sie aus dem Modell. Zuerst schliessen wir die post Kalter-Krieg Dummyvariable aus, dann religiöse Diversität, danach Demokratie, im Weiteren Polarisierung, anschließend ethnische Diversität und schliesslich das bergige Terrain. Somit erhalten wir unser Basismodell in Spalte 3 und seine Variante, in der wir den Anteil junger Männer in weiterführenden Schulen mit dem Pro-Kopf-Einkommen ersetzen, in Spalte 4. Weder weitere Reduzierungen noch Erweiterungen des Modelles sind zulässig. Das Basismodell und die Variante sind ähnlich in den Ergebnissen, obwohl der Erklärungsgehalt der Variante etwas geringer ist und zwei Variablen ihre Signifikanz verlieren (ethnische Dominanz und die geographische Verteilung der Bevölkerung).

Unser Basismodell erlaubt es uns, die Reaktion des Bürgerkriegsrisikos auf die Änderung der erklärenden Variablen zu schätzen. Wir stellen diese Schätzungen in Collier und Hoeffler (2001) dar. Wenn sich alle Variablen im Mittelwert befinden, beträgt das Bürgerkriegsrisiko 11.5 Prozent. Unser Modell sagt voraus, dass ein Land, das mit den schlechtesten Eigenschaften ausgestattet ist, mit fast 100-prozentiger Wahrscheinlichkeit einen Bürgerkrieg erleben wird, wohingegend ein Land, das mit den besten Eigenschaften ausgestattet ist, ein sehr sicheres Land ist. In Collier und Hoeffler (2001) bestimmen wir, wie die Veränderung einer Variablen das Bürgerkriegsrisiko beeinflußt (wir nehmen dabei an, daß sich alle anderen Variablen im Mittelwert befinden). Der Effekt von Primärproduktexporten auf das Bürgerkriegsrisiko ist relativ hoch. Bei dem Wert, der das Risiko maximiert (der Anteil der Primärproduktexporte beträgt dann 32 Prozent des BSP) liegt das Bürgerkriegsrisiko bei etwa 22 Prozent, während ein Land ohne solche Exporte ein Risiko von nur einem Prozent hat. Opportunitätskosten haben ebenfalls einen deutlichen Einfluss auf das Bürgerkriegsrisiko. Wenn der Anteil junger Männer, die weiterführende Schulen besuchen, um 10 Prozent ansteigt, ist das durchschnittliche Kriegsrisiko um drei Prozentpunkte reduziert (von 11.5 Prozent auf 8.6 Prozent). Ein Prozentpunkt

15 Wir benutzen die gleiche Stichprobe wie für das Kombinationsmodell $(n=665)$ und erhalten die folgenden Ergebnisse: Möglichkeitenmodell gegen Kombinationsmodell, 5 Freiheitsgrade, Likelihood Ratio Test (LRT) Statistik 7.85, p=0.165; Leid Modell gegen Kombinationsmodell, 6 Freiheitsgrade, LRT Statistik 29.64, p=0.000. 
zusätzliches Wachstum des Pro-Kopf-Einkommens reduziert das Risiko ebenfalls um etwa einen Prozentpunkt (von 11.5 Prozent auf 10.4 Prozent). Andere Variablen, welche die Kosten der Rebellion messen, sind ebenfalls signifikant. Direkt nach dem Ende eines Bürgerkrieges beträgt das Risiko eines erneuten Bürgerkriegsausbruches etwa 32 Prozent, dieses Risiko schwächt sich über die Zeit hinweg um etwa ein Prozent pro Jahr ab.

Die Variablen, die den relativen militärischen Vorteil der Rebellen messen sind ferner wichtig für die Höhe des Bürgerkriegsrisikos. Länder, in welchen die Bevölkerung stark konzentriert ist, haben ein sehr geringes Risiko wohingegen Länder mit einer zersiedelten Struktur ein höheres Risiko von etwa 37 Prozent besitzen. Dies unterstützt die Hypothese von Herbst (2000). Wie bereits besprochen, reduziert eine starke soziale Fragmentierung das Konfliktrisiko. Länder mit der maximalen sozialen Fragmentierung haben ein sehr geringes Risiko, es beträgt etwa ein Viertel des Risikos sozial homogener Länder. Allerdings hat ein Land, das durch ethnische Dominanz charakterisiert ist, ein doppelt so hohes Konfliktrisiko wie ein Land, in dem keine ethnische Gruppe dominiert. Der Effekt von Diversität ist somit eine Kombination des Fragmentierungs- und Dominanzeffektes. Mit zunehmender Vielfalt steigt das Risiko zunächst, nimmt dann aber ab. Daraus ließe sich schließen, dass Polarisierung wichtig ist, wir finden allerdings keinen statistisch signifikanten Zusammenhang zwischen Polarisierung und Konfliktrisiko.

\section{Robustheitsanalyse}

In diesem Abschnitt untersuchen wir die Robustheit unseres Basismodelles. Wir analysieren die Sensitivität der Ergebnisse im Hinblick auf Daten-Probleme und verschiedene Schätzmethoden. ${ }^{16}$ Mögliche Datenprobleme sind z.B. der Einfluss von Ausreißern und die unterschiedlichen Definitionen der abhängigen und unabhängigen Variablen. Im Hinblick auf die Schätzmethoden untersuchen wir Random-Effekts, Fixed-Effekts und einen Schätzer, der für seltene Ereignisse korrigiert.

Wir analysieren den Effekt von Ausreißern, indem wir überprüfen, ob unsere Ergebnisse sensitiv im Hinblick auf bestimmte Beobachtungen (influential data points) sind. Wir verwenden die Methode von Pregibon (1981), um herauszufinden, welche Beobachtungen einflussreich sind. Nach Ausschluss dieser Beobachtungen aus unserer Stichprobe untersuchen wir, ob sich unsere Ergebnisse verändern. Nach dieser Methode ergeben sich drei einflussreiche Beobachtungen. Schließen wir nun diese aus unserer Regression aus (Spalte 1), so

16 Weitere Robustheitstests werden in Collier und Hoeffler (2001) diskutiert. 
bleiben unsere Ergebnisse unverändert und der gesamte Erklärungswert verbessert sich (von $R^{2}=0.24$ auf $R^{2}=0.29$ ).

Tabelle 6: Robustheitstests

\begin{tabular}{|c|c|c|c|c|}
\hline & \multicolumn{4}{|c|}{ Logit Regressionen, Ereignis: Bürgerkriegsausbrüche } \\
\hline & $1^{17}$ & $2^{18}$ & $3^{19}$ & $4^{20}$ \\
\hline & $\begin{array}{l}\text { Ohne } \\
\text { einflußreiche } \\
\text { Beobachtungen }\end{array}$ & $\begin{array}{l}\text { Ohne hohe } \\
\text { Primärprodukt- } \\
\text { exporteure }\end{array}$ & $\begin{array}{l}\text { Friedensepisoden } \\
\text { von weniger als } \\
\text { einem Monat } \\
\text { werden als } \\
\text { durchgehender } \\
\text { Krieg definiert }\end{array}$ & $\begin{array}{l}\text { Friedensepisoden } \\
\text { von weniger als } \\
12 \text { Monaten } \\
\text { werden als } \\
\text { durchgehender } \\
\text { Krieg definiert }\end{array}$ \\
\hline $\begin{array}{l}\text { Schulbesuch } \\
\text { junger Männer }\end{array}$ & $\begin{array}{l}-0.041 \\
(0.011)^{* * * *}\end{array}$ & $\begin{array}{l}-0.031 \\
(0.010)^{* * * *}\end{array}$ & $\begin{array}{l}-0.031 \\
(0.010) * * *\end{array}$ & $\begin{array}{l}-0.031 \\
(0.010)^{* * *}\end{array}$ \\
\hline $\begin{array}{l}\text { Einkommens- } \\
\text { wachstum }\end{array}$ & $\begin{array}{l}-0.137 \\
(0.046)^{* * * *}\end{array}$ & $\begin{array}{l}-0.122 \\
(0.044) * * *\end{array}$ & $\begin{array}{l}-0.102 \\
(0.044) * * *\end{array}$ & $\begin{array}{l}-0.071 \\
(0.047)\end{array}$ \\
\hline $\begin{array}{l}\text { Primärprodukt- } \\
\text { exporte/BSP }\end{array}$ & $\begin{array}{l}28.745 \\
(7.862)^{* * * *}\end{array}$ & $\begin{array}{l}18.771 \\
(6.063)^{* * *}\end{array}$ & $\begin{array}{l}19.147 \\
(5.939)^{* * * *}\end{array}$ & $\begin{array}{l}22.686 \\
(6.718)^{* * * *}\end{array}$ \\
\hline $\begin{array}{l}\text { (Primärprodukt- } \\
\text { exporte/BSP) }\end{array}$ & $\begin{array}{l}-59.818 \\
(17.781) * * *\end{array}$ & $\begin{array}{l}-28.466 \\
(12.299) * *\end{array}$ & $\begin{array}{l}-30.150 \\
(12.031) * * *\end{array}$ & $\begin{array}{l}-39.053 \\
(14.405)^{* * * *}\end{array}$ \\
\hline $\begin{array}{l}\text { Soziale } \\
\text { Fragmentierung }\end{array}$ & $\begin{array}{l}-0.0003 \\
(0.0001) * * *\end{array}$ & $\begin{array}{l}-0.0002 \\
(0.0001) * *\end{array}$ & $\begin{array}{l}-0.0002 \\
(0.0001)^{* *}\end{array}$ & $\begin{array}{l}-0.0002 \\
(0.0001)^{* * * *}\end{array}$ \\
\hline $\begin{array}{l}\text { Ethnische } \\
\text { Dominanz }\end{array}$ & $\begin{array}{l}0.655 \\
(0.372)^{*}\end{array}$ & $\begin{array}{l}0.647 \\
(0.354)^{*}\end{array}$ & $\begin{array}{l}0.732 \\
(0.357)^{* *}\end{array}$ & $\begin{array}{l}0.741 \\
(0.362)^{* *}\end{array}$ \\
\hline Friedensperiode & $\begin{array}{l}-0.004 \\
(0.0011)\end{array}$ & $\begin{array}{l}-0.004 \\
(0.001)^{* * * *}\end{array}$ & $\begin{array}{l}-0.003 \\
(0.001)^{* * * *}\end{array}$ & $\begin{array}{l}-0.003 \\
(0.001)^{* * * *}\end{array}$ \\
\hline $\ln$ Bevölkerung & $\begin{array}{l}0.899 \\
(0.195)^{* * * *}\end{array}$ & $\begin{array}{l}0.772 \\
(0.168) * * *\end{array}$ & $\begin{array}{l}0.782 \\
(0.167) * * *\end{array}$ & $\begin{array}{l}0.832 \\
(0.176) * * *\end{array}$ \\
\hline $\begin{array}{l}\text { Verteilung der } \\
\text { Bevölkerung }\end{array}$ & $\begin{array}{l}-2.890 \\
(1.136)^{* * * *}\end{array}$ & $\begin{array}{l}-2.449 \\
(1.008) * *\end{array}$ & $\begin{array}{l}-2.541 \\
(1.012) * * *\end{array}$ & $\begin{array}{l}-2.953 \\
(1.049) * * *\end{array}$ \\
\hline $\mathrm{N}$ & 685 & 662 & 686 & 683 \\
\hline $\begin{array}{l}\text { Anzahl der } \\
\text { Bürgerkriegs- } \\
\text { ausbrüche }\end{array}$ & 43 & 46 & 44 & 41 \\
\hline Pseudo $\mathrm{R}^{2}$ & 0.29 & 0.21 & 0.23 & 0.21 \\
\hline Log likelihood & -114.04 & -122.23 & -126.33 & -122.23 \\
\hline
\end{tabular}

Bemerkungen: Alle Regressionen haben eine Konstante. Standardfehler in Klammern, p indiziert das Signifikanzniveau. ***,**, * indizieren Signifikanz auf dem 1, 5 and 10 Prozent-Niveau.

17 Wir schließen die folgenden drei einflussreichen Beobachtungen aus: Iran 1970-74, Rumänien 1985-89, Kongo 1995-99.

18 Wir schließen die Länder mit dem höchsten Anteil von Primärproduktexport am BSP aus: Saudi Arabien, Guyana, Oman und Trinidad und Tobago. Ihr durchschnittlicher Anteil ist 0.504 (Mittelwert der Stichprobe ist 0.158).

19 Wir schließen die folgenden Kriegsausbrüche aus: Angola 1975 und Somalia 1988.

20 Wir schließen die folgenden Kriegsausbrüche aus: Angola 1975, Mosambik 1976, Sierra Leone 1997, Somalia 1988 und Zaire/DRC 1997. 
Wir untersuchen weiterhin, ob friedliche Länder mit sehr hohen Anteilen von Primärproduktexporten für den nicht-monotonen Effekt auf das Bürgerkriegsrisiko verantwortlich sind. Vier Länder, die nicht durch Bürgerkriegsepisoden gekennzeichnet sind, haben besonders hohe Anteile. Spalte 2 in Tabelle 6 zeigt die Ergebnisse unseres Basismodelles unter Ausschluss dieser Länder. Die Ergebnisse sind weitgehend unverändert, der nicht-monotone Zusammenhang zwischen Primärproduktexporten und Bürgerkriegsrisiko wird bestätigt.

Nun wenden wir uns der Frage der Variablen-Definition zu. Der umstrittenste Aspekt der abhängigen Variablen ist die Unterscheidung zwischen einem langen Bürgerkrieg oder multiplen Bürgerkriegen, die durch kurze Friedensperioden unterbrochen sind. In der Analyse haben wir uns soweit auf die Definitionen der Politikwissenschaflter verlassen, die die Datensätze erstellten. Einige Friedensperioden zwischen zwei Bürgerkriegsepisoden sind allerdings recht kurz, und es kann sein, daß es besser ist, diese Kriege als einen fortlaufenden Bürgerkrieg aufzufassen. Wir definieren daher Kriege, die durch sehr kurze Friedensperioden von weniger als einem Monat gekennzeichnet sind, als einen fortlaufenden Krieg. Diese Ergebnisse sind in Spalte 3 dargestellt, das Basismodell ist weitgehend unverändert. Wenn wir Friedensperioden von weniger als 12 Monaten vernachlässigen (Spalte 4), erhalten wir ähnliche Ergebnisse, allerdings ist der Koeffizient der Wachstumsrate nun marginal insignifikant $(\mathrm{p}=0.12)$.

Wir untersuchen auch unterschiedliche Definitionen von ethnischer Dominanz und sozialer Fragmentierung. Unsere Definition von ethnischer Dominanz, 45 bis 90 Prozent der Bevölkerung gehören einer ethnischen Gruppe an, ist kompatibel mit theoretischen Modellen, welche die Ausbeutung von Minderheiten erklären (siehe Collier, 2001). Andere Definitionen, wie z.B. der Anteil der größten ethnischen Gruppe an der Gesamtbevölkerung sind statistisch nicht signifikant. Wir experimentierten auch mit anderen Definitionen sozialer Fragmentierung, aber finden ebenfalls keine Aggregation von ethnischer und religiöser Diversität, die unsere Ergebnisse dominieren. Zudem berechneten wir den Polarisationsindex für unterschiedliche Werte für $\alpha$, aber in allen Fällen war die Polarisation insignifikant. Die Analyse einer alternative Polarisationsvariable (Reynal-Querol, 2001) und der Anzahl der ethnischen Gruppen zeigte ebenfalls, dass diese Variablen nicht signifikant sind.

In Tabelle 7 analysieren wir verschiedene Schätzmethoden. Wir schätzen unser Basismodell mit der Random-Effekts Methode und zeigen, dass die Ergebnisse sich nicht statistisch von denen der gepoolten Regression unterscheiden, d.h. wir akzeptieren die Hypothese, dass wir über die Beobachtungen hinweg poolen 
können. ${ }^{21}$ Die Schätzung des Fixed-Effekt Logit war nur für eine sehr kleine Stichprobe möglich, da nur Länder berücksichtigt werden können, die sowohl Friedens- wie auch Kriegsepisoden haben. Obwohl die Stichprobe damit drastisch reduziert wird, bleibt der nicht-monotone Effekt der Primärproduktexporte erhalten. Wenn dieser Effekt nur in der gepoolten Schätzung auftreten würde, könnte man vermuten, dass es sich hier um die Approximation eines Geographieeffekts handeln könnte. Da dies nicht der Fall ist, sind wir uns sicher, dass wir andere Erklärungen des Primärprodukteffekts ausschliessen können.

Möglichen Zeiteffekte tragen wir Rechnung indem wir binäre Zeitvariablen in das Modell aufnehmen. Aufgrund eines Likelihood Ratio Testes können wir die Hypothese, dass alle diese Dummy-Variablen null sind, nicht ablehnen. ${ }^{22}$

$21 \quad$ Ein LRT produzierte die Teststatistik $\chi \quad{ }^{2}=0 \quad(\mathrm{p}=0.998)$, d.h. wir können die Nullhypothese, dass der Panel Schätzer und der gepoolte Schätzer gleich sind, nicht ablehnen.

22 Die LRT Statistik ist $\chi^{2}=7.83,6$ Restriktionen, $\mathrm{p}=0.251$. 
Tabelle 7: Schätzmethoden

\begin{tabular}{|c|c|c|c|c|}
\hline & \multicolumn{4}{|c|}{ Logit Regressionen, Ereignis: Bürgerkriegsausbrüche } \\
\hline & 1 & 2 & 3 & 4 \\
\hline & $\begin{array}{l}\text { Random } \\
\text { Effekts }\end{array}$ & $\begin{array}{l}\text { Fixed } \\
\text { Effekts }\end{array}$ & $\begin{array}{l}\text { Pooled Logit } \\
\text { Zeit- } \\
\text { Dummies }\end{array}$ & $\begin{array}{l}\text { Seltene } \\
\text { Ereignisse } \\
\text { Logit }\end{array}$ \\
\hline Schulbesuch junger Männer & $\begin{array}{l}-0.032 \\
(0.010)^{* * * *}\end{array}$ & $\begin{array}{l}0.007 \\
(0.033)\end{array}$ & $\begin{array}{l}-0.031 \\
(0.010)^{* * * *}\end{array}$ & $\begin{array}{l}-0.029 \\
(0.010)^{* * *}\end{array}$ \\
\hline Einkommenswachstum & $\begin{array}{l}-0.115 \\
(0.043) * * *\end{array}$ & $\begin{array}{l}-0.045 \\
(0.072)\end{array}$ & $\begin{array}{l}-0.129 \\
(0.047) * * *\end{array}$ & $\begin{array}{l}-0.110 \\
(0.040)^{* * *}\end{array}$ \\
\hline Primärproduktexporte/BSP & $\begin{array}{l}18.937 \\
(5.865)^{* * * *}\end{array}$ & $\begin{array}{l}35.850 \\
(14.436) * * *\end{array}$ & $\begin{array}{l}18.895 \\
(5.988)^{* * * *}\end{array}$ & $\begin{array}{l}17.161 \\
(6.535)^{* * * *}\end{array}$ \\
\hline (Primärproduktexporte/BSP) ${ }^{2}$ & $\begin{array}{l}-29.443 \\
(11.782)^{* * * *}\end{array}$ & $\begin{array}{l}-65.967 \\
(26.964) * * *\end{array}$ & $\begin{array}{l}-29.815 \\
(12.098) * * *\end{array}$ & $\begin{array}{l}-25.594 \\
(14.355)^{*}\end{array}$ \\
\hline Soziale Fragmentierung & $\begin{array}{l}-0.0002 \\
(0.0001)^{* *}\end{array}$ & $\begin{array}{l}-0.007 \\
(0.006)\end{array}$ & $\begin{array}{l}-0.0002 \\
(0.0001) * *\end{array}$ & $\begin{array}{l}-0.0002 \\
(0.0001)^{* *}\end{array}$ \\
\hline $\begin{array}{l}\text { Ethnische Dominanz } \\
(45-90 \%)\end{array}$ & $\begin{array}{l}0.670 \\
(0.354)^{*}\end{array}$ & & $\begin{array}{l}0.682 \\
(0.359) * \\
\end{array}$ & $\begin{array}{l}0.644 \\
(0.336)^{*}\end{array}$ \\
\hline Friedensperiode & $\begin{array}{l}-0.004 \\
(0.001)^{* * *}\end{array}$ & $\begin{array}{l}0.011 \\
(0.002)^{* * * *}\end{array}$ & $\begin{array}{l}-0.004 \\
(0.001)^{* * *}\end{array}$ & $\begin{array}{l}-0.004 \\
(0.001)^{* * *}\end{array}$ \\
\hline Ln Bevölkerung & $\begin{array}{l}0.768 \\
(0.166) * * *\end{array}$ & $\begin{array}{l}0.010 \\
(1.410) \\
\end{array}$ & $\begin{array}{l}0.762 \\
(0.170)^{* * * *}\end{array}$ & $\begin{array}{l}0.726 \\
(0.151)^{* * * *}\end{array}$ \\
\hline Verteilung der Bevölkerung & $\begin{array}{l}-2.487 \\
(1.005)^{* * * *}\end{array}$ & $\begin{array}{l}115.363 \\
(74.562)\end{array}$ & $\begin{array}{l}-2.447 \\
(1.018) * *\end{array}$ & $\begin{array}{l}-2.394 \\
(1.085)^{* *}\end{array}$ \\
\hline $\mathrm{T} 70-74$ & & & $\begin{array}{l}0.725 \\
(0.602) \\
\end{array}$ & \\
\hline T75-79 & & & $\begin{array}{l}0.578 \\
(0.608) \\
\end{array}$ & \\
\hline $\mathrm{T} 80-84$ & & & $\begin{array}{l}1.137 \\
(0.602)^{*}\end{array}$ & \\
\hline T85-89 & & & $\begin{array}{l}-0.013 \\
(0.757)\end{array}$ & \\
\hline T90-94 & & & $\begin{array}{l}0.802 \\
(0.677)\end{array}$ & \\
\hline T95-99 & & & $\begin{array}{l}-0.492 \\
(0.921)\end{array}$ & \\
\hline $\mathrm{N}$ & 688 & 145 & 688 & 688 \\
\hline $\begin{array}{l}\text { Anzahl der } \\
\text { Bürgerkriegsausbrüche }\end{array}$ & 46 & 44 & 46 & \\
\hline Pseudo $\mathrm{R}^{2}$ & & & 0.26 & \\
\hline Log likelihood & -128.21 & -38.18 & -124.30 & \\
\hline
\end{tabular}

Bemerkungen: Alle Regressionen haben eine Konstante. Standardfehler in Klammern. ***, **, * indizieren Signifikanz auf dem 1, 5 and 10 Prozent-Niveau.

Als letzten Robustheitstest benutzen wir eine neue Methode zur Korrektur von seltenen Ereignissen (King und Zen, 2001). Unser Datensatz ist durch eine relativ geringe Anzahl von Ereignissen gekennzeichnet, da nur etwa sieben Prozent unserer Beobachtungen Bürgerkriegsausbrüche darstellen. King und Zen (2001) zeigen, dass in solchen Situationen die Standard-Logit-Ergebnisse die Wahrscheinlichkeit eines Ereignisses oft signifikant unterschätzen. Wir benutzen 
ihren Korrekturmechanismus, die hieraus resultierenden neuen Ergebnisse unterscheiden sich nur unwesentlich von denen des Standard-Logit-Schätzers (Spalte 4).

Wir experimentierten ebenfalls mit einer Reihe von anderen ModellSpezifikationen. Wir fanden keine der folgenden geographischen und demographischen Charakteristika signifikant: Bewaldung, Bevölkerungsdichte und Urbanisierungsgrad. ${ }^{23}$

\section{Interpretation und Schlussfolgerung}

Wir untersuchen das Risiko eines Bürgerkriegsausbruches mit Hilfe eines globalen Datensatzes, der den Zeitraum von 1960 bis 1999 umfasst. In dieser Untersuchung benutzen wir Logit Regressionen, um das Risiko eines Kriegsausbruches in den folgenden fünf Jahren zu analysieren. Wir testen zwei unterschiedliche Erklärungsansätze und finden, dass Modelle, welche die Möglichkeiten der Rebellen untersuchen, dieses Risiko besser erklären als Modelle, die sich auf eine Theorie von Leid induzierten Rebellionen stützen. Unser Modell ist robust in Bezug auf Ausreißer, verschiedene Variablendefinitionen und alternative Spezifikationen.

Der leichtverständlichste Aspekt der Theorie der Möglichkeiten ist die Finanzierung der Rebellion, die nötig ist, um den Kampf gegen die Regierung zu ermöglichen. Wir zeigen, dass Länder mit hohem Vorkommen an natürlichen Ressourcen, gemessen als Anteil der Primärproduktexporte am BSP, ein erhöhtes Risiko von Bürgerkriegsausbrüchen haben. Die Ausbeutung von natürlichen Ressourcen kann sehr lukrativ sein und stellt eine ausgezeichnete Finanzquelle oder sogar einen Anreiz zur Rebellion dar. Andererseits sind Länder mit vielen natürlichen Ressourcen oft durch schlechte Regierungen und Institutionen charakterisiert, die möglicherweise Leid in der Bevölkerung verursachen. Daher könnten natürliche Ressourcen auch anders interpretiert werden. Wir kontrollieren aber für andere Faktoren, die die wirtschaftliche Leistungsfähigkeit erfassen, z.B. das Niveau, das Wachstum und die Verteilung des Einkommens sowie politische Rechte. Eine große Anzahl von Fallstudien zeigt ebenfalls, dass natürliche Ressourcen eine Finanzierungsmöglichkeit für Rebellionen darstellen. Fallstudien indizieren weiterhin, dass Diasporen in erheblichem Maße helfen, Bürgerkriege in ihren Heimatsländern zu finanzieren. Wir definieren Diasporen als den Anteil der Bevölkerung, der im Ausland lebt. Wir finden, dass Länder mit größerer Diaspora

23 Der Urbanisierungsgrad ist signifikant, wenn wir die geographische Verteilung der Bevölkerung nicht berücksichtigen. Wenn wir beide Variablen im Modell verwenden, ist die geographische Verteilung der Bevölkerung statistisch signifikant, wohingegen der Urbanisierungsgrad marginal insignifikant ist $(\mathrm{p}=0.11)$. 
ein höheres Bürgerkriegsrisiko haben. Es ist schwer, sich eine andere Interpretation vorzustellen, als die Theorie der besseren Finanzierungsmöglichkeiten durch die Diaspora.

Ein zweiter Aspekt der Möglichkeiten ist ein erhöhtes Risiko in Situationen, in denen Rebellion preiswert ist, oder anders ausgedrückt, je niedriger die Opportunitätskosten sind, desto einfacher ist es, Rebellen zu rekrutieren und eine Rebellion zu organisieren. Wir erfassen Opportunitätskosten mit dem Anteil junger Männern, die weiterführende Schule besuchen, mit dem Pro-KopfEinkommen und der Wachstumsrate der vorangegangenen fünf Jahre. Alle Variablen sind statistisch hoch signifikant. Man könnte niedrige Einkommen allerdings auch als Ursache von Leid interpretieren. In diesem Fall müsste aber auch die Einkommensverteilung ein Risikofaktor sein, wir finden allerdings hierfür keinen empirischen Hinweis.

Ein dritter Aspekt der Möglichkeiten-Theorie sind militärische Vorteile. Wir finden, dass eine weit verstreute Bevölkerung und bergiges Terrain das Bürgerkriegsrisiko erhöhen.

Eine Reihe von Leidfaktoren waren insignifikant: Ungleichheit, politische Rechte, ethnische Polarisierung und religiöse Fragmentierung. Nur ethnische Dominanz, wenn eine ethnische Gruppe die Mehrheit stellt, hat einen adversen Effekt auf das Bürgerkriegsrisiko. Dies muss man im Zusammenhang mit dem positiven Effekt von sozialer Fragmentierung auf das Bürgerkriegsrisiko sehen. Ein höherer Grad von ethnischer und religiöser Diversität stabilisiert ein Land. Wir nehmen an, dass es schwieriger ist, Kohäsion in einer Rebellenorganisation zu erreichen, wenn Rebellen von diversen ethnischen und religiösen Gruppen rekrutiert werden müssen.

Das Bürgerkriegsrisiko erhöht sich proportional mit der Gesamtbevölkerung des Landes. Daher nehmen wir an, dass die Möglichkeiten proportional mit der Größe der Bevölkerung steigen, es aber weniger plausibel ist, dass Leid von der Größe der Bevölkerung abhängt.

Eine Variable, die Friedensperiode seit dem letzten Bürgerkrieg, kann als Möglichkeiten- oder als Leidfaktor interpretiert werden. Sie kann die graduelle Abdiskontierung von konflikt-spezifischem Kapital und somit die steigenden Kosten einer neuen Rebellion erfassen, oder sie kann mit dem abnehmenden Hass korreliert sein.

Wir zeigen, dass Möglichkeiten einen Erklärungsansatz darstellen, der mit der ökonomischen Interpretation konsistent ist, dass Rebellionen von Gier motiviert werden. Dies bedeutet aber nicht, dass die Rebellen gewöhnliche Kriminelle sind, Leidfaktoren können eine Rolle in der Motivation der Rebellen spielen. Allerdings zeigen unsere Ergebnisse, dass diese Leidfaktoren wahrscheinlich nicht mit den sozialen Problemen von Ungleichheit, politischen Rechten und ethnisch-religiöser Identität verbunden sind. 


\section{Literaturverzeichnis}

Alesina, A., S. Ötzler, N. Roubini und P. Swagel. 1996. 'Political Instability and Economic Growth.' Journal of Economic Growth 1:189-211.

Alesina, A., R. Baqir und W. Easterly. 1999. ' Public Goods and Ethnic Divisions.' Quarterly Journal of Economics 114:1243-84.

Angoustures, A. und V. Pascal. 1996. 'Diasporas et financement des conflits,' in Jean, F. und J. C. Rufin (Hrg.) Economie des Geurres Civiles, Hachette, Paris.

Barro, R. J. 1991. 'Economic Growth in a Cross Section of Countries.' The Quarterly Journal of Economics 106:407-43.

Barrett, D. B. (Hrg.). 1982. World Christian Encyclopedia. Oxford: Oxford University Press.

Buchanan, J. M. und R .L. Faith. 1987. 'Secession and the Limits of Taxation: Towards a Theory of Internal Exit.' American Economic Review, 77:1023-31.

Collier, P. 2001. Ethnic diversity: An economic analysis of its implications.' Economic Policy (angenommen).

Collier, P. und A. Hoeffler. 1998. 'On the Economic Causes of Civil War.' Oxford Economic Papers, 50:563-73.

-------. 2001. ' Greed and Grievance in Civil War' , CEPR Working Paper, angenommen (http://www.cepr.org)

Collier, P., A. Hoeffler und M. Söderbom. 2001. 'On the Duration of Civil.' World Bank Policy Reseach Working Paper 2681, Washington D.C..

Davidson R. und J. G. MacKinnon. 1981. 'Several Tests for model specification in the presence of alternative hypotheses.' Econometrica 49:781-793.

Deininger, K. und L. Squire. 1996. A New Data Set Measuring Income Inequality. World Bank Economic Review 10:565-91.

-------1998. 'New Ways of Looking at Old Issues: Inequality and Growth.' Journal of Development Economics 57:249-287.

Easterly, W. und R. Levine. 1997. 'Africa's Growth Tragedy: Policies and Ethnic Divisions.' Quarterly Journal of Economics 113:1203-49.

Esteban, J.-M. und D. Ray. 1994. 'On the Measurement of Polarization.' Econometrica 62(4): 819-51.

-1999. 'Conflict and Distribution.' Journal of Economic Theory 87:379415. 
Figes, O. 1996. 'A People's Tragedy: The Russian Revolution 1891-1924', London, Pimlico.

Gerrard, A. J. W. 2000. 'What is a Mountain?' Unveröffentlichtes Manuskript. DECRG, World Bank.

Grossman, H. I. 1991. A General Equilibrium Model of Insurrections. American Economic Review 81:912-21.

1999. Kleptrocracy and Revolutions. Oxford Economic Papers 51:267283.

Hegre, H., T. Ellingsen, S. Gates, and N.-P. Gleditsch. 2001. 'Toward a Democratic Civil Peace? Democracy, Political Change, and Civil War, 1816-1992'. American Political Science Review 95:33-48.

Herbst, J. 2000. 'States and Power in Africa'. Princeton. Princeton University Press.

Hirshleifer, J. 1995. 'Theorizing about Conflict'. In: Handbook of Defense Economics, K. Hartley and T. Sandler (Hrg.), 165-89. Vol. 1. Amsterdam: Elsevier Science.

--------. 2001. 'The Dark Side of the Force: Economic Foundations of Conflict Theory'. Cambridge, UK: Cambridge University Press.

Jaggers, K. und T. R. Gurr. 1995. 'Tracking Democracy's Third Wave with the Polity III Data'. Journal of Peace Research 32:469-482.

King, G. und L. Zeng. 2001. 'Logistic Regression in Rare Events Data'. Political Analysis, angenommen.

Klare, M.T. 2001. Natural Resource Wars: The New Landscape of Global Conflict, Metropolitan Books, New York.

Mauro, P. 1995. 'Corruption and Growth'. The Quarterly Journal of Economics 110:681-712.

Pregibon, D. 1981. Logistic Regression Diagnostics. The Annals of Statistics 9:705-24.

Reynal-Querol, M. 2001. 'Ethnicity, Political Systems and Civil War'. Journal of Conflict Resolution, forthcoming.

Sachs, J. D. und A.M. Warner. 1995. 'Natural Resource Abundance and Economic Growth'. National Bureau of Economic Research Working Paper: 5398.

2000. Natural Resource Abundance and Economic Growth. In: Leading Issues in Economic Development. 7th Aufl., G. M. Meier and J. E. Rauch (Hrg.). Oxford: Oxford University Press.

Sen, A. 1973. On economic inequality. Oxford: Clarendon Press. 
Singer, D. J. und M. Small. 1994. Correlates of War Project: International and Civil War Data, 1816-1992. Inter-University Consortium for Political and Social Research, Ann Arbor, Michigan (Datensatz).

Small, M. und J. D. Singer. 1982. 'Resort to Arms: International and Civil War, 1816-1980’. Beverly Hills: Sage.

The Stockholm International Peace Research Institute. 2001. Yearbook of World Armaments and Disarmaments. Oxford: Oxford University Press.

Summers, R. und A. Heston. 1991. 'The Penn World Table (Mark 5): An Expanded Set of International Comparisons, 1950-1988'. The Quarterly Journal of Economics 99:327-68.

USSR. 1964. Atlas Narodov Mira, Department of Geodesy and Cartography of the State Geological Committee of the USSR. Moscow.

World Bank. 2000. World Development Indicators, Washington DC (Datensatz). 


\section{Anhang: Variablendefinitionen und Datenquellen24}

\section{Bergiges Terrain}

Der Anteil des Landes, der bergig ist, wurde für uns von John Gerrard bestimmt, die Daten werden in Gerrard (2000) besprochen.

\section{Bevölkerung}

Bevölkerung misst die Gesamtbevölkerung. Datenquelle: Weltbank World Development Indicators 1998.

\section{Bewaldung}

Wir benutzen die FAO Messwerte für den Anteil des Landes, der mit Wäldern bedeckt ist, Datenquelle: http://www.fao.org/forestry

\section{Bürgerkriege}

Wir verwenden hauptsächlich die Daten von Singer und Small $(1984,1994)$ und wenden ihre Daten für interne und extra-systemische Kriege an. Wir möchten uns bei Nicholas Sambanis, Mitarbeiter der Weltbank, für seine Hilfe mit der Erweiterung dieser Datensätze bedanken.

\section{Ethnische Dominanz}

Wir benutzen Daten über ethno-linguistische Zugehörigkeit von der Originalquelle (Atlas Narodov Mira, 1964), um unsere ethnische Dominanz-Variable zu erstellen. Sie nimmt den Wert von eins an, wenn 45 bis 90 Prozent der Bevölkerung einer ethnischen Gruppe zugehören. Wir möchten uns bei Tomila Lankina für ihre Forschungsassistenz im Zusammenhang mit der Übersetzung der Daten bedanken.

24 Eine detaillierte Diskussion der Datenquellen findet sich in Collier und Hoeffler (2001). 


\section{Friedensperiode}

Diese Variable erfasst die Länge der Friedensperiode in Monaten seit dem Ende des letzten Bürgerkrieges, oder seit dem Ende des zweiten Weltkrieges falls das Land nie einen Bürgerkrieg erlebte.

\section{Geographische Verteilung der Bevölkerung}

Wir konstruieren einen Gini-Koeffizienten der Bevölkerung, der auf Bevölkerungszahlen für $400 \mathrm{~km}^{2}$ Zellen beruht, d.h. ein Wert von eins bedeutet, dass die Bevölkerung völlig homogen über das Land verstreut ist und ein Wert von null, dass die gesamte Bevölkerung in einer Zelle konzentriert ist. Wir möchten uns bei Uwe Deichman, Mitarbeiter der Weltbank (World Bank's Geographic Information System Unit), für seine Hilfe mit diesen Daten bedanken.

\section{Primärproduktexporte/BSP}

Wir approximieren den Ressourcenreichtum eines Landes durch den Anteil der Primärproduktexporte am Bruttosozialprodukt. Die Daten wurden von der Weltbank zur Verfügung gestellt; wir folgen der Definition von Sachs und Warner (1995, 2000).

\section{Pro-Kopf-Einkommen}

Das Pro-Kopf-Einkommen wird in konstanten und der Kaufkraftparität angepassten US Dollars gemessen. Die Daten wurden den Penn World Tables 5.6 (Summers und Heston, 1991) entnommen. Da die Daten nur für 1960-92 verfügbar sind, verwenden wir die Wachstumsraten der Weltbank um das ProKopf-Einkommen für die Neunziger Jahre zu errechnen.

\section{Soziale, ethnische und religiöse Fragmentierung}

Wir approximieren die soziale Fragmentierung in einem kombinierten Index basierend auf ethnischer und religiöser Fragmentierung. Ethnische Fragmentierung wird durch den ethno-linguistischen Fraktionalisations-Index gemessen. Er mißt die Wahrscheinlichkeit, dass zwei zufällig herausgegriffene Menschen in einem gegebenen Land nicht die gleiche Sprache sprechen. Diese Daten sind nur für den Anfang der Sechziger Jahre erhältlich; in der volkswirtschaftlichen Literatur wurde dieser Index zum ersten Mal von Mauro (1995) verwendet. Wir benutzen Daten über Religionszugehörigkeiten von Barrett (1982), um einen analogen religiösen Fragmentierungsindex zu errechnen. Ein 
Wert von null indiziert eine völlig homogene Gesellschaft und ein Wert von 100 würde eine völlig heterogene Gesellschaft beschreiben. Wir kalkulieren unseren sozialen Fragmentierungsindex als das Produkt von ethno-linguistischer Fragmentierung und religiöser Fragmentierung plus entweder dem ethnolinguistischen oder religiösen Fragmentierung-Index. Wir addieren hierbei immer den größeren Index. Damit vermeiden wir, dass ein Land als homogen klassifiziert wird (ein Wert von null), wenn entweder die ethnische oder religiöse Fragmentierung null ist, aber nicht homogen in Bezug auf die andere Variable ist. 